\title{
Resummed heavy quark production cross sections to next-to-leading logarithm
}

\author{
N. Kidonakis \\ Department of Physics and Astronomy, University of Edinburgh, Edinburgh EH9 3JZ, Scotland \\ J. Smith \\ Deutsches Electronen-Synchrotron, DESY, Notkestrasse 85, D-22603, Hamburg, Germany \\ and Institute for Theoretical Physics, State University of New York at Stony Brook, Stony Brook, New York 11794-3840 \\ R. Vogt \\ Nuclear Science Division, Lawrence Berkeley National Laboratory, Berkeley, California 94720 \\ and Department of Physics, University of California at Davis, Davis, California 95616
}

(Received 16 August 1996; revised manuscript received 3 February 1997)

\begin{abstract}
We study how next-to-leading logarithms modify predictions from leading logarithmic soft-gluon resummation in the heavy quark production cross section near threshold. Numerical results are presented for top quark production at the Fermilab Tevatron and bottom quark production at fixed-target energies. [S0556-2821(97)01815-8]

PACS number(s): 12.38.Cy, 13.85.Lg, 14.65.Fy, 14.65.Ha
\end{abstract}

\section{INTRODUCTION}

The calculation of hadronic cross sections in the elastic limit (i.e., near threshold) in perturbative QCD involves contributions from the emission of soft gluons. In $n$th order QCD one encounters leading logarithmic (LL) contributions proportional to $\left(-\alpha_{s}^{n} / n !\right)\left\{\ln ^{2 n-1}\left[(1-z)^{-1}\right] /(1-z)\right\}_{+}$. There are also terms with next-to-leading logarithmic (NLL) contributions proportional to $\left(-\alpha_{s}^{n} / n !\right)\left\{\ln ^{2 n-2}\left[(1-z)^{-1}\right] /\right.$ $(1-z)\}_{+}$. These "plus" distributions are large near threshold, $z=1$ (the precise definition of $z$ will be given in the next section) and resummation techniques were originally developed to sum them in Drell-Yan production [1]. As the DrellYan process involves electroweak interactions, it has a rather simple color structure. Gluons are only radiated from the incoming quark-antiquark pair in the hadronic collision process so the amplitude is a color singlet.

The first resummation of LL terms (as well as some NLL terms) in the heavy (top) quark production cross section was discussed $[2,3]$ prior to the discovery of the top quark at the Fermilab Tevatron [4]. The analysis was based on the fact that the LL terms are identical to those in the Drell-Yan process and, even though a cutoff was used to define the resummed perturbation series, this paper pointed out the importance of incorporating resummation effects in threshold production of heavy quarks in QCD. Recently other groups have applied more sophisticated LL resummation methods to the top quark production cross section, see Refs. [5,6], which avoid the use of an explicit cutoff and lead to slightly different values for the top quark cross section. At present the experimental results cannot discriminate between them and there is an ongoing discussion as to which method is theoretically superior. The quark-antiquark annihilation channel is the dominant partonic process for top quark production in $p \bar{p}$ collisions at center-of-mass energy $\sqrt{S}=1.8 \mathrm{TeV}$. However, the gluon-gluon channel is more important in bottom and charm production near threshold in fixed-target experi- ments with proton and pion beams. The LL resummation method of [2] has also been applied to $b$-quark production at HERA-B [7] and more generally for fixed-target bottom and charm production by hadron beams [8].

The order $\alpha_{s}^{2}$ corrections to the Drell-Yan process in [9] have allowed a check of the NLL terms in the resummation formulas, thus the theory is in excellent shape. However, this information cannot be used in heavy-quark resummation since some of the NLL terms are a consequence of the more complicated color structure in heavy quark production. There is no exact calculation of the heavy quark production cross section at order $\alpha_{s}^{4}$. Even though the NLL terms at order $\alpha_{s}^{3}$ for heavy quark production near threshold were available in 1990 [10], at the time of the heavy quark resummation analysis [2] it was not clear how to resum them. Therefore all work has concentrated on the LL terms. Now this situation has changed. A resummation formalism which correctly incorporates NLL resummation near threshold has recently been presented by Kidonakis and Sterman [11,12]. The authors analytically compared the order-by-order expansion of their results with the known NLL terms [10] and found agreement at threshold in both the quark-antiquark channel and the gluon-gluon channel.

In this paper we will apply this new NLL resummation formalism to calculate the top quark production cross section at the Fermilab Tevatron and the bottom quark production cross section at fixed target energies. We are particularly interested in the size and therefore the phenomenological importance of the NLL terms with respect to the LL terms. To determine the relative importance of the NLL terms, we will use the cutoff method proposed originally in [2]. There are several reasons for this, which we now discuss. First, as already stated, we are primarily interested in the corrections the NLL terms make to the LL terms. Next, by varying the cutoff the failure of the perturbative expansion and the onset of the nonperturbative region may be studied directly, bypassed in the newer methods. Finally, given the complexity of the formulas it is best to use the simplest approach to 
incorporate the NLL terms in a first test of their magnitude. An analysis of the NLL terms is very important since the present LL resummations are based either on neglecting them entirely [5] or retaining only some of them [6]. These terms are not universal and could possibly be of importance in several areas of perturbative QCD besides the analysis of the heavy quark cross section, including the production of large transverse energy jets and the production of supersymmetric particles. The conclusions of our study should not be generalized to these other reactions since each process is different and requires a separate study.

\section{GENERAL FORMALISM}

Here we present some basic formulas which we need for our analysis, based on the theory developed by Kidonakis and Sterman $[11,12]$. For heavy quark production to be kinematically allowed, the square of the partonic center-ofmass energy $s=x_{a} x_{b} S$ must be larger than the threshold value of the invariant mass of the heavy quark-antiquark pair $Q^{2}=4 m^{2}$. When the heavy quarks are produced with zero velocity, the true threshold and the partonic threshold are equivalent. In either case, the plus distributions which must be resummed are functions of

$$
z=\frac{Q^{2}}{s} .
$$

Our calculations are based on the factorization of soft gluons from high-energy partons in perturbative QCD $[13,14]$. All the singular distributions in the heavy quark $(Q)$ production cross section can be expressed in the form

$$
\begin{aligned}
& \frac{d \sigma_{h_{1} h_{2}}}{d Q^{2} d \cos \theta^{*} d y} \\
& =\sum_{a b} \sum_{I J} \int \frac{d x_{a}}{x_{a}} \frac{d x_{b}}{x_{b}} \phi_{a / h_{1}}\left(x_{a}, Q^{2}\right) \phi_{b / h_{2}}\left(x_{b}, Q^{2}\right) \\
& \quad \times \delta\left(y-\frac{1}{2} \ln \frac{x_{a}}{x_{b}}\right) \hat{\sigma}_{a b}^{(I J)}\left(\frac{Q^{2}}{x_{a} x_{b} S}, \theta^{*}, \alpha_{s}\left(Q^{2}\right)\right),
\end{aligned}
$$

where $x_{a}, x_{b}$ are the momentum fractions of the incoming partons, $y$ is the rapidity of the $Q \bar{Q}$ pair, and $\theta^{*}$ is the scattering angle in the pair center-of-mass system. The $\phi$ 's are parton densities, evaluated at the mass factorization scale $Q^{2}$, in the deep inelastic scattering (DIS) or modified minimal subtraction scheme $\overline{\mathrm{MS}}$ mass factorization scheme. The hard scattering functions $\hat{\sigma}$, which depend on the color structure $(I J)$ of the interaction as well as on the partonic channel $a b$ and the mass factorization scheme, contain all the plus distributions in the threshold region. In [11] it was shown that up to NLL it is possible to pick a color basis in which moments of the functions $\hat{\sigma}_{a b}^{(I J)}\left(z, \theta^{*}, \alpha_{s}\left(Q^{2}\right)\right)$ exponentiate with respect to $z$ so that

$$
\begin{aligned}
\widetilde{\sigma}_{a b}^{(I J)}\left(n, \theta^{*}, Q^{2}\right) & =\int_{0}^{1} d z z^{n-1} \hat{\sigma}_{a b}^{(I J)}\left(z, \theta^{*}, \alpha_{s}\left(Q^{2}\right)\right) \\
& =h_{I}\left(\theta^{*}, Q^{2}\right) h_{J}^{*}\left(\theta^{*}, Q^{2}\right) e^{E_{I J}\left(n, \theta^{*}, Q^{2}\right)} .
\end{aligned}
$$

The hard scattering prefactor is a product of the contributions from the amplitude $h_{I}$ and its complex conjugate $h_{J}^{*}$. Therefore $I$ refers to the decomposition of the hard scattering amplitude into a color basis, e.g., into an $s$-channel singlet and octet. The functions $h_{I}$ and $h_{J}^{*}$ have no collinear or soft divergences at the partonic threshold since these terms have been factored into the exponent $E_{I J}$ in Eq. (2.3). The exponential in Eq. (2.3), called $S_{I J}$ in [11,12], satisfies a renormalization group equation with an anomalous dimension ma$\operatorname{trix} \Gamma_{I J}$.

The exponents of Eq. (2.3) are given by

$$
\begin{aligned}
E_{I J}^{(a b)}\left(n, \theta^{*}, Q^{2}\right)= & -\int_{0}^{1} d z \frac{z^{n-1}-1}{1-z}\left\{(2-r) \int_{0}^{z} \frac{d y}{1-y}\right. \\
& \times g_{1}^{(a b)}\left\{\alpha_{s}\left[(1-y)^{2-r}(1-z)^{r} Q^{2}\right]\right\} \\
& +g_{2}^{(a b)}\left\{\alpha_{s}\left[(1-z) Q^{2}\right]\right\} \\
& +g_{3}^{(I)}\left\{\alpha_{s}\left[(1-z)^{2} Q^{2}\right], \theta^{*}\right\} \\
& \left.+g_{3}^{(J) *}\left\{\alpha_{s}\left[(1-z)^{2} Q^{2}\right], \theta^{*}\right\}\right\}, \quad
\end{aligned}
$$

where $g_{1}, g_{2}$, and $g_{3}$ are functions of the running coupling constant $\alpha_{s}$ with $z$-dependent (and $y$-dependent) arguments. The parameter $r, 1$ in the DIS scheme and 0 in the $\overline{\mathrm{MS}}$ scheme, effectively changes the lower limit of the $y$ integral in the $\overline{\mathrm{MS}}$ scheme (see [15] and our later discussion). As it stands, Eq. (2.4) is ill defined since it incorporates arbitrarily soft gluon radiation at the point where the QCD perturbation expansion diverges. There are different opinions as to how to define the exponent in a manner which clearly separates the perturbative and nonperturbative regions. We will return to this point shortly.

To go to NLL in the exponents $E_{I J}$, we need $g_{1}$ up to and including $O\left(\alpha_{s}^{2}\right)$ and $g_{2}$ and $g_{3}^{(I)}$ to $O\left(\alpha_{s}\right)$. References [5,6] do not include the NLL $g_{3}$ term, treated numerically for the first time here.

The functions $g_{1}$ and $g_{2}$ depend on the mass factorization scheme and the identity of the incoming partons but are essentially independent of the color structure. The function $g_{1}$ is scheme dependent because of the $r$ dependence of the $y$ integration. It also depends on the identity of the incoming partons so that

$$
g_{1}^{(a b)}\left[\alpha_{s}\right]=\left(C_{a}+C_{b}\right)\left[\frac{\alpha_{s}}{\pi}+\frac{1}{2} K\left(\frac{\alpha_{s}}{\pi}\right)^{2}\right],
$$

where $C_{q}=C_{q}=C_{F}$ and $C_{g}=C_{A}$. The constant $K$ is (cf. [16])

$$
K=C_{A}\left(\frac{67}{18}-\frac{\pi^{2}}{6}\right)-\frac{5}{9} n_{\mathrm{f}},
$$


where $n_{\mathrm{f}}$ is the number of quark flavors. The $g_{2}$ contribution is only nonzero in the DIS scheme [15]:

$$
g_{2}^{(q \bar{q}) \mathrm{DIS}}\left[\alpha_{s}\right]=-\frac{3}{2} C_{F} \frac{\alpha_{s}}{\pi} .
$$

Otherwise, $g_{2}^{(q \bar{q})} \overline{\mathrm{MS}}=g_{2}^{g g}=0$.

The functions $g_{3}^{(I)}$ depend on the color structure in the hard scattering but are mass factorization scheme independent. In [11,12] it is shown that they are the eigenvalues of the anomalous dimension matrix $\Gamma_{I J}$ which appears in the renormalization group equation for the function $S_{I J}$. The precise relation between the $g_{3}^{(I)}$ and the eigenvalues of $\Gamma_{I J}$ is

$$
g_{3}^{(I)}\left\{\alpha_{s}\left[(1-z)^{2} Q^{2}\right], \theta^{*}\right\}=-\lambda_{I}\left\{\alpha_{s}\left[(1-z)^{2} Q^{2}\right], \theta^{*}\right\},
$$

showing that $g_{3}^{(I)}$ and $\lambda_{I}$ are functions of both the running coupling constant $\alpha_{s}$ (evaluated at a $z$-dependent scale) and the angle $\theta^{*}$ between the directions of the incoming parton and outgoing heavy quark. As can be seen below, although the eigenvalues are complex, the total $g_{3}$ contribution to $E_{I J}^{(a b)}$ in Eq. (2.4) is real.

Consider first the reaction channel $q\left(p_{a}\right)+\bar{q}\left(p_{b}\right)$ $\rightarrow \bar{Q}\left(p_{i}\right)+Q\left(p_{j}\right)$, where $\Gamma_{I J}$ is two dimensional. In the $s$-channel singlet-octet basis the color decomposition is into $\delta^{a b} \delta^{i j}$ (singlet) and $-\delta^{a b} \delta^{i j} /(2 N)+\delta^{a j} \delta^{b i} / 2$ (octet). In this basis, the components of the matrix $\Gamma_{I J}$ are [12]

$$
\begin{gathered}
\Gamma_{11}=-\frac{\alpha_{s}}{\pi} C_{F}\left(L_{\beta}+1+i \pi\right), \\
\Gamma_{21}=\frac{2 \alpha_{s}}{\pi} \ln \left(\frac{u_{1}}{t_{1}}\right), \\
\Gamma_{12}=\frac{\alpha_{s}}{\pi} \frac{C_{F}}{C_{A}} \ln \left(\frac{u_{1}}{t_{1}}\right), \\
\Gamma_{22}=\frac{\alpha_{s}}{\pi}\left\{C_{F}\left[4 \ln \left(\frac{u_{1}}{t_{1}}\right)-L_{\beta}-1-i \pi\right]+\frac{C_{A}}{2}\left[-3 \ln \left(\frac{u_{1}}{t_{1}}\right)\right.\right. \\
\left.\left.-\ln \left(\frac{m^{2} s}{t_{1} u_{1}}\right)+L_{\beta}+i \pi\right]\right\},
\end{gathered}
$$

where

$$
L_{\beta}=\frac{1-2 m^{2} / s}{\beta}\left[\ln \left(\frac{1-\beta}{1+\beta}\right)+i \pi\right]
$$

and $\beta^{2}=1-4 \mathrm{~m}^{2} / \mathrm{s}$. The Mandelstam invariants for the reaction are $s=\left(p_{a}+p_{b}\right)^{2}, \quad t=t_{1}+m^{2}=\left(p_{a}-p_{i}\right)^{2}, \quad$ and $u=u_{1}+m^{2}=\left(p_{b}-p_{i}\right)^{2}$.

$\Gamma_{I J}$ is diagonalized in this basis when the parton-parton c.m. scattering angle $\theta^{*}=90^{\circ} \quad\left(u_{1}=t_{1}=-s / 2\right.$ at threshold $)$ with eigenvalues

$$
\begin{gathered}
\lambda_{1}=\lambda_{\text {singlet }}=-\frac{\alpha_{s}}{\pi} C_{F}\left(L_{\beta}+1+i \pi\right), \\
\lambda_{2}=\lambda_{\text {octet }}=\frac{\alpha_{s}}{\pi}\left\{-C_{F}\left(L_{\beta}+1+i \pi\right)\right. \\
\left.+\frac{C_{A}}{2}\left[L_{\beta}-\ln \left(\frac{m^{2} s}{t_{1}^{2}}\right)+i \pi\right]\right\} .
\end{gathered}
$$

It is also diagonalized at partonic threshold $s=4 \mathrm{~m}^{2}$ for arbitrary $\theta^{*}$.

In the partonic channel $g\left(p_{a}\right)+g\left(p_{b}\right) \rightarrow \bar{Q}\left(p_{i}\right)+Q\left(p_{j}\right)$ the anomalous dimension matrix is three dimensional. In the color basis $\delta^{a b} \delta_{j i}, d^{a b c} T_{j i}^{c}$, and $i f^{a b c} T_{j i}^{c}$ the components of the matrix $\Gamma_{I J}$ are [12]

$$
\begin{gathered}
\Gamma_{11}=-\frac{\alpha_{s}}{\pi}\left[C_{F}\left(L_{\beta}+1\right)+C_{A} i \pi\right], \\
\Gamma_{21}=0, \\
\Gamma_{31}=\frac{2 \alpha_{s}}{\pi} \ln \left(\frac{u_{1}}{t_{1}}\right), \\
\Gamma_{12}=0, \\
\Gamma_{22}=\frac{\alpha_{s}}{\pi}\left\{-C_{F}\left(L_{\beta}+1\right)+\frac{C_{A}}{2}\left[-\ln \left(\frac{m^{2} s}{t_{1} u_{1}}\right)+L_{\beta}-i \pi\right]\right\}, \\
\Gamma_{32}=\frac{N^{2}-4}{4 N} \Gamma_{31}, \\
\Gamma_{13}=\frac{1}{2} \Gamma_{31}, \\
\Gamma_{23}=\frac{C_{A}}{4} \Gamma_{31}, \\
\Gamma_{33}=\Gamma_{22} .
\end{gathered}
$$

At $\theta^{*}=90^{\circ}$ the anomalous dimension matrix becomes diagonal with eigenvalues

$$
\begin{gathered}
\lambda_{1}=-\frac{\alpha_{s}}{\pi}\left[C_{F}\left(L_{\beta}+1\right)+C_{A} i \pi\right], \\
\lambda_{2}=\frac{\alpha_{s}}{\pi}\left\{-C_{F}\left(L_{\beta}+1\right)+\frac{C_{A}}{2}\left[L_{\beta}-\ln \left(\frac{m^{2} s}{t_{1}^{2}}\right)-i \pi\right]\right\}, \\
\lambda_{3}=\lambda_{2} .
\end{gathered}
$$

We note that $\Gamma_{I J}$ is also diagonal at partonic threshold for arbitrary $\theta^{*}$.

\section{HEAVY-QUARK PRODUCTION CROSS SECTIONS}

As we have already stated, we are primarily interested in the magnitude of the NLL terms, particularly the $g_{3}$ contribution, relative to the LL results of $[2,3,7,8]$. Therefore we use a similar cutoff scheme, modifying the definitions of the 
exponents in Eqs. (2.3) and (2.4) to work directly in momentum space and avoid working in Mellin space. This method, introduced in [2], exploits the correspondence between the moment variable $n$ and logarithmic terms in the momentum space variable $1-z$. To directly compare with other recent LL resummation schemes in Refs. $[5,6]$ would, in principle, require three separate studies since each group of authors defines Eq. (2.4) differently. Therefore, in this paper we only consider the approach based on [2].

First, we identify

$$
1-z=\frac{s_{4}}{2 m^{2}}
$$

where $s_{4}$ is the invariant mass of the heavy-quark + gluon system which recoils against the detected heavy antiquark in inclusive $\bar{Q}$ production. The invariants satisfy $s+t_{1}+u_{1}=s_{4}$ so that in the elastic limit $s_{4} \rightarrow 0$ or $z \rightarrow 1$. Note that Eq. (3.1) differs from the identification $1-z=s_{4} / m^{2}$ in Refs. [2,5]. The factor of 2 is necessary to compare the NLL terms [12] with the expressions previously given in [10]. With the identification in Eq. (3.1) all the NLL terms in the $q \bar{q}$ channel are reproduced, not only exactly at threshold but also at finite $s_{4}$ except for one NLL term in $C_{F}^{2}$ which has a different coefficient. (This small difference is due to the fact that the $Q \bar{Q}$ invariant mass and the $Q g$ invariant mass are different invariants.) A similar observation holds for the $g g$ channel.

Further we exploit the fact that a heavy quark-antiquark pair will be preferentially produced back-to-back in the parton-parton center-of-mass frame near threshold. We also choose the case of $\theta^{*}=90^{\circ}$ to use the results of Eqs. (2.11) and (2.13). This choice avoids both the diagonalization of the three-dimensional matrix in the gluon-gluon channel and an extra numerical integration over $\theta^{*}$.

The resummation is done using Eqs. (3.17) and (3.19) of [2] where the "plus" distributions are removed through integration by parts. Therefore the resummed partonic cross section is defined as

$$
\sigma_{a b}\left(s, m^{2}\right)=-\int_{s_{\mathrm{cut}}}^{s-2 m s^{1 / 2}} d s_{4} f_{a b}\left(\frac{s_{4}}{2 m^{2}}\right) \frac{d \bar{\sigma}_{a b}^{(0)}\left(s, s_{4}, m^{2}\right)}{d s_{4}}
$$

where $a b=q \bar{q}$ or $g g$ and $s_{\text {cut }}$ will be defined below. The other inputs needed here are the function $f_{a b}$, in either the DIS scheme or the $\overline{\mathrm{MS}}$ scheme and the differential of the Born cross section. The exponential function $f_{a b}\left(s_{4} / 2 m^{2}\right)$ is given by a momentum space version of Eq. (2.4). This is best illustrated by examining Eqs. (3.31) and (3.35) in [2]. There we showed how to include gluons with an invariant mass $s_{4}^{\prime}>s_{4}$ and integrate over their transverse momenta up to the kinematic limit. The corresponding result follows by first replacing $z$ in Eq. (2.4) by $z^{\prime}$. Next we replace $z^{\prime n-1}-1$ in the numerator of Eq. (2.4), by -1 and introduce the variables $\omega^{\prime}=1-z^{\prime}=s_{4}^{\prime} /\left(2 m^{2}\right)$ and $\xi=(1-y)\left(1-z^{\prime}\right) Q^{2} / \Lambda^{2}$. By explicitly leaving $Q^{2}$ in the following discussion, we can later study the scale dependence of the calculation. Note that $\alpha_{s}$ in Eqs. (2.5)-(2.13) is a function of $s_{4}^{\prime} / 2 m^{2}$.
We first discuss the $q \bar{q}$ channel. There is only one kinematic structure in this channel so that the singlet and octet eigenvalues in Eq. (2.11) multiply the same function. At this order in perturbation theory, however, only the octet component contributes, see the discussion in [11]. Therefore in the DIS scheme at $\theta^{*}=90^{\circ}$, the exponential function is

$$
f_{q \bar{q}}^{\mathrm{DIS}}\left(\frac{s_{4}}{2 m^{2}}\right)=\exp \left[E_{q \bar{q}}^{\mathrm{DIS}}+E_{q \bar{q}}\left(\lambda_{2}\right)\right]
$$

where

$$
\begin{aligned}
E_{q \bar{q}}^{\mathrm{DIS}}= & E_{q \bar{q}}^{\mathrm{DIS}}\left(g_{1}\right)+E_{q \bar{q}}^{\mathrm{DIS}}\left(g_{2}\right) \\
= & \int_{\omega_{0}}^{1} \frac{d \omega^{\prime}}{\omega^{\prime}}\left\{\int_{\omega^{\prime 2} Q^{2} / \Lambda^{2}}^{\omega^{\prime} Q^{2} / \Lambda^{2}} \frac{d \xi}{\xi}\left[\frac{2 C_{F}}{\pi}\left(\alpha_{s}(\xi)+\frac{1}{2 \pi} \alpha_{s}^{2}(\xi) K\right)\right]\right. \\
& \left.-\frac{3}{2} \frac{C_{F}}{\pi} \alpha_{s}\left(\frac{\omega^{\prime} Q^{2}}{\Lambda^{2}}\right)\right\} .
\end{aligned}
$$

Since our calculation is not done in moment space, the $\omega^{\prime}$ integral is cut off at $\omega_{0}=s_{4} / 2 m^{2}$. Because the running coupling constant diverges when $\omega^{\prime 2} Q^{2} / \Lambda^{2} \sim 1$, the minimum cutoff in Eq. (3.2) is $s_{\text {cut }}=s_{4, \min } \sim 2 m^{2} \Lambda / Q$. If we choose the scale $Q^{2}=m^{2}, s_{\text {cut }} \sim 2 m \Lambda$. In general we choose a larger value to stay away from the point of divergence. Once we have chosen a reasonable cutoff, consistent with the sum of the first few terms in the perturbative expansion, we can study the size of the NLL terms with respect to the LL terms (the order-by-order expansion does not require a cutoff).

We use the two-loop running coupling constant

$$
\begin{gathered}
\alpha_{s}(\xi)=\frac{1}{a \ln \xi}+\frac{b}{a} \frac{\ln (\ln \xi)}{\ln ^{2}(\xi)}, \\
a=\frac{11 C_{A}-4 T_{\mathrm{f}} n_{\mathrm{f}}}{12 \pi}, \\
b=-6 \frac{17 C_{A}^{2}-\left(6 C_{F}+10 C_{A}\right) T_{\mathrm{f}} n_{\mathrm{f}}}{\left(11 C_{A}-4 T_{\mathrm{f}} n_{\mathrm{f}}\right)^{2}},
\end{gathered}
$$

with $C_{A}=3, C_{F}=4 / 3$ and $T_{\mathrm{f}}=1 / 2$. It is obvious that when $\xi<1, \ln \xi$ is negative so that $\ln (\ln \xi)$ has a cut and needs a precise definition. We use the cutoff on the $\omega^{\prime}$ variable to stop the integration before that point. The exact cutoff depends on the quark mass, the scale and $\Lambda_{n_{\mathrm{f}}}$, the QCD scale parameter for $n_{\mathrm{f}}$ light quark flavors, in the parton densities. Both the $g_{1}$ integrals over $\omega^{\prime}$ and $\xi$ and the $g_{2}$ integral over $\omega^{\prime}$ as well as the contributions to the $g_{3}$ integration which only contain $s_{4}^{\prime}$ in the running coupling constant can be done analytically, as shown in the Appendix.

In the $\overline{\mathrm{MS}}$ scheme,

$$
f_{q \bar{q}}^{\overline{\mathrm{MS}}}\left(\frac{s_{4}}{2 m^{2}}\right)=\exp \left[E_{q \bar{q}}^{\overline{\mathrm{MS}}}+E_{q \bar{q}}\left(\lambda_{2}\right)\right]
$$

where now 


$$
\begin{aligned}
E_{q \bar{q}}^{\overline{\mathrm{MS}}}= & E_{q \bar{q}}\left(g_{1}\right)=\int_{\omega_{0}}^{1} \frac{d \omega^{\prime}}{\omega^{\prime}} \\
& \times \int_{\omega^{\prime 2} Q^{2} / \Lambda^{2}}^{Q^{2} / \Lambda^{2}} \frac{d \xi}{\xi}\left[\frac{2 C_{F}}{\pi}\left(\alpha_{s}(\xi)+\frac{1}{2 \pi} \alpha_{s}^{2}(\xi) K\right)\right] .
\end{aligned}
$$

Note the difference between the upper limits of the $\xi$ integrations introduced by the mass factorization scheme in Eqs. (3.4) and (3.7).

The color-dependent $g_{3}$ contribution in Eq. (2.11) leads to

$$
\begin{aligned}
E_{q \bar{q}}\left(\lambda_{i}\right)= & -\int_{\omega_{0}}^{1} \frac{d \omega^{\prime}}{\omega^{\prime}}\left\{\lambda_{i}\left[\alpha_{s}\left(\frac{\omega^{\prime 2} Q^{2}}{\Lambda^{2}}\right), \theta^{*}=90^{\circ}\right]\right. \\
& \left.+\lambda_{i}^{*}\left[\alpha_{s}\left(\frac{\omega^{\prime 2} Q^{2}}{\Lambda^{2}}\right), \theta^{*}=90^{\circ}\right]\right\}
\end{aligned}
$$

in both mass factorization schemes, where $i=1,2$.

The differential function in Eq. (3.2) follows from the kinematic behavior of the Born cross section, represented by

$$
F_{q \bar{q}}^{B}\left(s, t_{1}, u_{1}\right)=\frac{t_{1}^{2}+u_{1}^{2}}{s^{2}}+\frac{2 m^{2}}{s} .
$$

Substituting $t_{1}=-1 / 2\left\{s-s_{4}-\left[\left(s-s_{4}\right)^{2}-4 s m^{2}\right]^{1 / 2} \cos \theta^{*}\right\}$ and $u_{1}=-1 / 2\left\{s-s_{4}+\left[\left(s-s_{4}\right)^{2}-4 s m^{2}\right]^{1 / 2} \cos \theta^{*}\right\}$, and taking $\theta^{*}=90^{\circ}$, we define, analogous to Eq. (2.20) in [2],

$$
\bar{F}_{q \bar{q}}^{(0)}=\frac{\left[\left(s-s_{4}\right)^{2}-4 s m^{2}\right]^{1 / 2}}{2 s^{2}} F_{q \bar{q}}^{B} .
$$

Differentiating with respect to $s_{4}$, we find

$$
\frac{d \bar{F}_{q \bar{q}}^{(0)}}{d s_{4}}=-\frac{1}{4 s^{4}} \frac{s-s_{4}}{\sqrt{\left(s-s_{4}\right)^{2}-4 s m^{2}}}\left[3\left(s-s_{4}\right)^{2}-4 s m^{2}\right] .
$$

The integrand in Eq. (3.2) becomes, for both factorization schemes,

$$
\begin{aligned}
f_{q \bar{q}}\left(\frac{s_{4}}{2 m^{2}}\right) \frac{d \bar{\sigma}_{q \bar{q}}^{(0)}\left(s, s_{4}, m^{2}\right)}{d s_{4}}= & \pi \alpha_{s}^{2} K_{q \bar{q}} N C_{F} \frac{d \bar{F}_{q \bar{q}}^{(0)}}{d s_{4}} \\
& \times \exp \left[E_{q \bar{q}}+E_{q \bar{q}}\left(\lambda_{2}\right)\right],
\end{aligned}
$$

where $K_{q} \bar{q}=1 / N^{2}$ is a color average factor. We note that near threshold $d \bar{\sigma}_{q}^{(0)} / d s_{4}$ is approximately a factor of 2 smaller than $d \sigma_{q}^{(0)} / d s_{4}$ in Eq. (3.20) of Ref. [2] where the angle $\theta^{*}$ was analytically integrated before differentiating with respect to $s_{4}$.

The treatment of the gluon-gluon channel is very similar but now we have three distinct color structures. However, only two of them are independent. Therefore we define $f_{g g}$
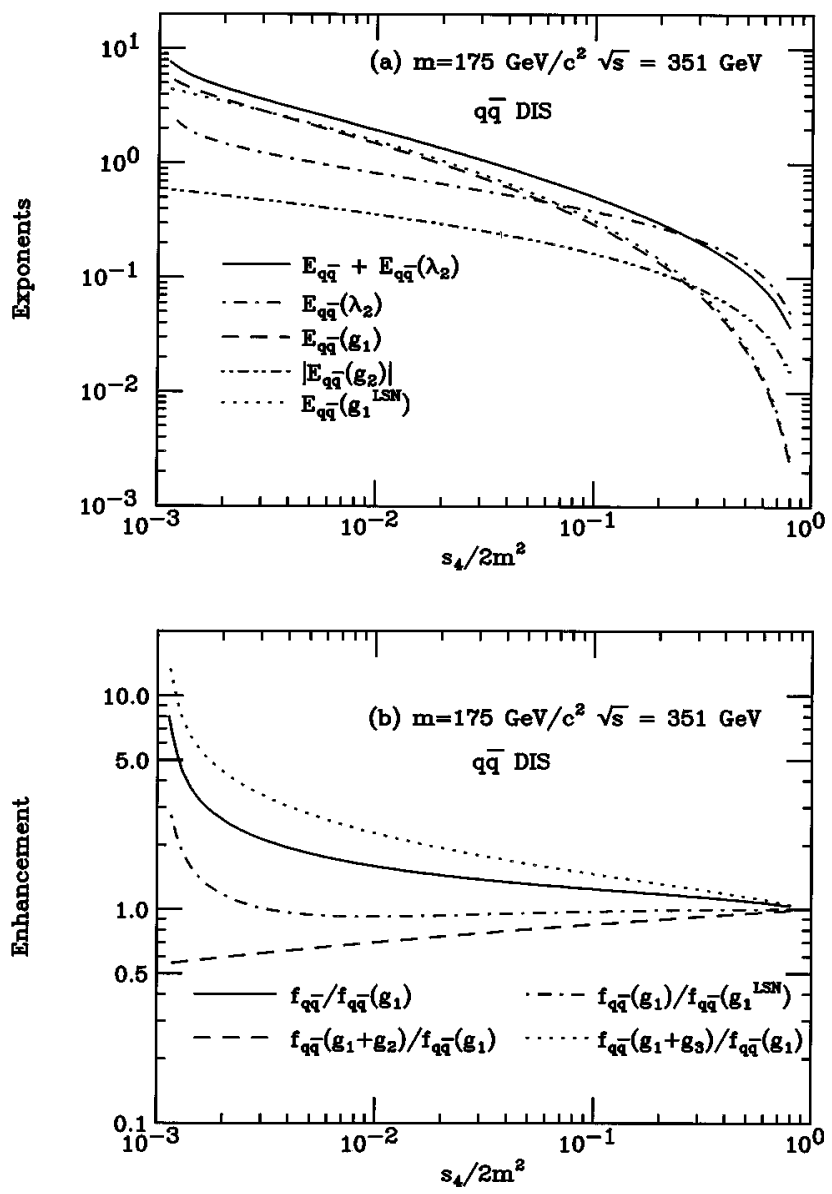

FIG. 1. (a) We show the contributions to $f_{q \bar{q}}^{\mathrm{DIS}}$ in top quark production for $m=175 \mathrm{GeV} / c^{2}$ and $\sqrt{s}=351 \mathrm{GeV}$ as a function of $s_{4} / 2 m^{2}$. The solid curve shows $\ln \left(f_{q \bar{q}}^{\mathrm{DIS}}\right)$ for the octet component at $90^{\circ}$, the dot-dashed curve $E_{q \bar{q}}\left(\lambda_{2}\right)$, the dashed curve $E_{q \bar{q}}^{\mathrm{DIS}}\left(g_{1}\right)$, and the dot-dot-dashed curve $\left|E_{q \bar{q}}^{\mathrm{DIS}}\left(g_{2}\right)\right|$. The dotted curve shows $E^{\text {DIS }}$ as defined in Eq. (3.30) of Ref. [2]. (b) The ratios of the exponential factors are given to show the enhancement relative to $f_{q \bar{q}}\left(g_{1}\right)$, where $f_{q} \bar{q}\left(g_{1}\right) \equiv \exp \left[E_{q \bar{q}}^{\mathrm{DIS}}\left(g_{1}\right)\right]$. The solid curve shows $f_{q \bar{q}}^{\mathrm{DIS}} / f_{q \bar{q}}-\left(g_{1}\right)$, the dashed curve $f_{q} \bar{q}\left(g_{1}+g_{2}\right) / f_{q \bar{q}}\left(g_{1}\right)$, and the dotted curve $f_{q} \bar{q}\left(g_{1}+g_{3}\left(\lambda_{2}\right)\right) / f_{q} \bar{q}\left(g_{1}\right)$. The ratio $f_{q} \bar{q}\left(g_{1}\right) / \exp \left(E^{\mathrm{DIS}}\right)$, given in the dot-dashed curve, shows the enhancement of $g_{1}$ from Eq. (2.5) relative to the one-loop value in [2].

for each eigenvalue so that $f_{g g, i}=\exp \left[E_{g g}+E_{g g}\left(\lambda_{i}\right)\right]$ with a one-to-one correspondence between $E_{g g}\left(\lambda_{i}\right)$ and the eigenvalues of Eq. (2.13) so that

$$
\begin{aligned}
E_{g g}\left(\lambda_{i}\right)= & -\int_{\omega_{0}}^{1} \frac{d \omega^{\prime}}{\omega^{\prime}}\left\{\lambda_{i}\left[\alpha_{s}\left(\frac{\omega^{\prime 2} Q^{2}}{\Lambda^{2}}\right), \theta^{*}=90^{\circ}\right]\right. \\
& \left.+\lambda_{i}^{*}\left[\alpha_{s}\left(\frac{\omega^{\prime 2} Q^{2}}{\Lambda^{2}}\right), \theta^{*}=90^{\circ}\right]\right\},
\end{aligned}
$$

where $i=1,2$. In the $\overline{\mathrm{MS}}$ scheme, we take

$$
E_{g g}=\int_{\omega_{0}}^{1} \frac{d \omega^{\prime}}{\omega^{\prime}} \int_{\omega^{\prime 2} Q^{2} / \Lambda^{2}}^{Q^{2} / \Lambda^{2}} \frac{d \xi}{\xi}\left[\frac{2 C_{A}}{\pi}\left(\alpha_{s}(\xi)+\frac{1}{2 \pi} \alpha_{s}^{2}(\xi) K\right)\right],
$$



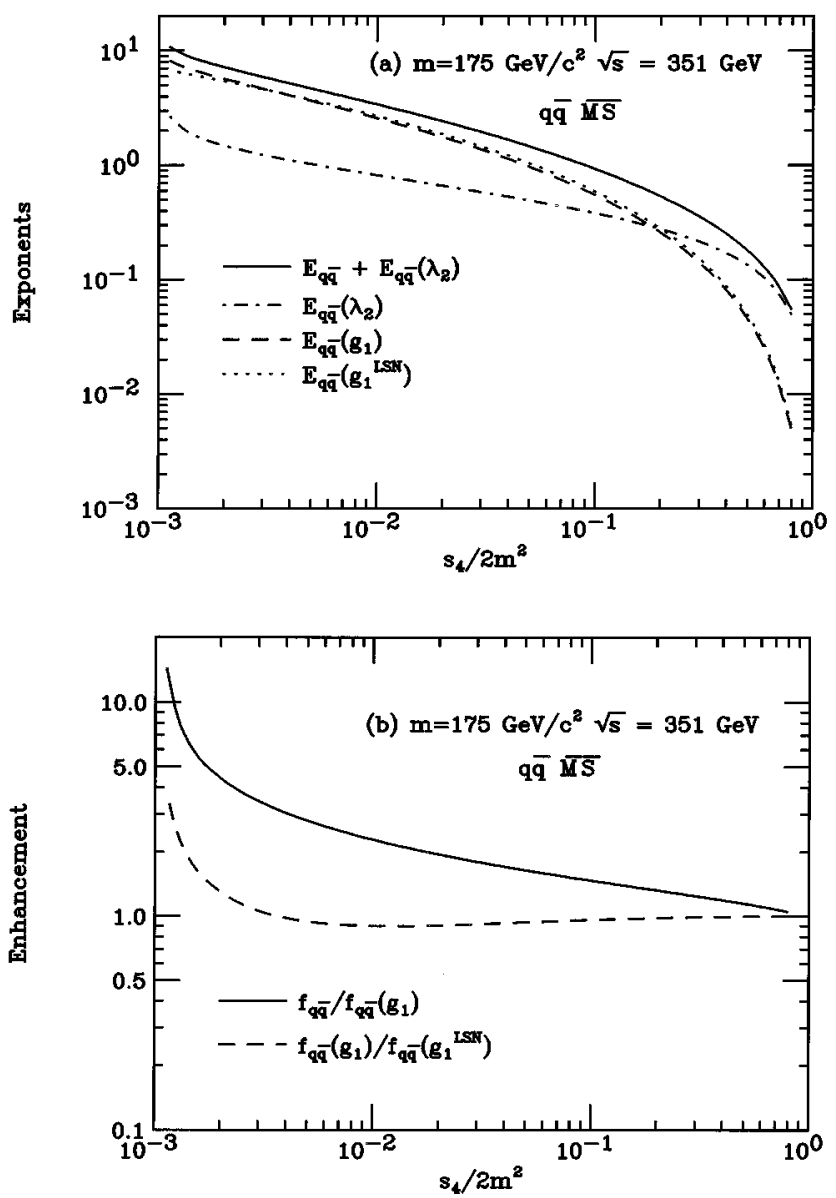

FIG. 2. (a) We show the contributions to $f_{q \bar{q}}^{\overline{\mathrm{MS}}}$ in top quark production for $m=175 \mathrm{GeV} / c^{2}$ and $\sqrt{s}=351 \mathrm{GeV}$ as a function of $s_{4} / 2 m^{2}$. The solid curve shows $\ln \left(f_{q \bar{q}}^{\overline{\mathrm{MS}}}\right)$ for the octet component at $90^{\circ}$, the dot-dashed curve $E_{q}-\left(\lambda_{2}\right)$, and the dashed curve $E_{q \bar{q}}^{\overline{\mathrm{MS}}}\left(g_{1}\right)$. The dotted curve shows $E^{\overline{\mathrm{MS}}}$ as defined in Eq. (3.34) of Ref. [2]. (b) The ratios of the exponential factors are given to show the enhancements. The solid curve shows $f_{q \bar{q}}^{\overline{\mathrm{MS}}} / f_{q \bar{q}}\left(g_{1}\right)$ while the dashed curve is the ratio $f_{q \bar{q}}\left(g_{1}\right) / \exp \left(E^{\overline{\mathrm{MS}}}\right)$, where $f_{q} \bar{q}\left(g_{1}\right) \equiv \exp \left[E_{q \bar{q}}^{\overline{\mathrm{MS}}}\left(g_{1}\right)\right]$.

where $K$ is defined as in Eq. (2.6). This choice of $K$ requires some justification, which we have not found explicitly in the literature, due to the fact that the discussions of the corrections to the Drell-Yan process are made in the DIS scheme. However, it should be possible to prove rigorously that Eq. (3.14) holds [17].

Because there are two kinematical structures, $d \bar{\sigma}_{g g}^{(0)} / d s_{4}$ has two contributions:

$$
\begin{aligned}
& F_{g g, I}^{B}\left(s, t_{1}, u_{1}\right)=\frac{t_{1}}{u_{1}}+\frac{u_{1}}{t_{1}}+\frac{4 m^{2} s}{t_{1} u_{1}}\left(1-\frac{m^{2} s}{t_{1} u_{1}}\right), \\
& F_{g g, I I}^{B}\left(s, t_{1}, u_{1}\right)=\left(1-\frac{4 t_{1} u_{1}}{s^{2}}\right) F_{g g, I}^{B}\left(s, t_{1}, u_{1}\right) .
\end{aligned}
$$

The second kinematical structure, proportional to the first, comes from the non-Abelian part of the cross section. As in Eq. (3.10), we define
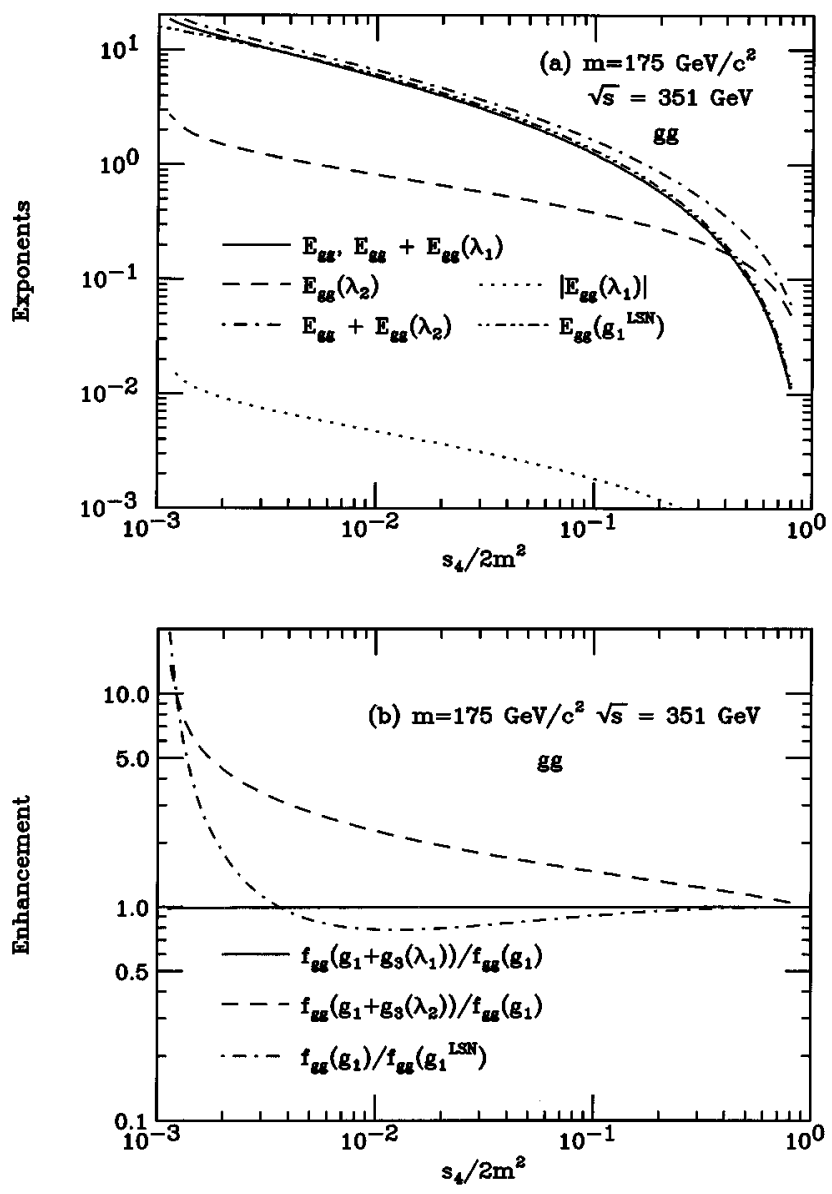

FIG. 3. (a) We show the contributions to $f_{g g}$ in top quark production for $m=175 \mathrm{GeV} / c^{2}$ and $\sqrt{s}=351 \mathrm{GeV}$ as a function of $s_{4} / 2 \mathrm{~m}^{2}$. The solid curve shows $E_{g g}$, the dashed and dotted curves give the contributions from the eigenvalues $E_{g g}\left(\lambda_{2}\right)$ and $\left|E_{g g}\left(\lambda_{1}\right)\right|$, respectively. The dot-dashed curve shows the sum $E_{g g}+E_{g g}\left(\lambda_{2}\right)$; the sum $E_{g g}+E_{g g}\left(\lambda_{1}\right)$ is indistinguishable from the solid curve. The dot-dot-dashed curve shows $E^{\overline{\mathrm{MS}}}$ for the gluon from Ref. [2]. (b) The ratios of the exponential factors are given to show the enhancements relative to $f_{g g}\left(g_{1}\right) \equiv \exp \left(E_{g g}\right)$. The solid curve shows $f_{g g}\left(g_{1}+g_{3}\left(\lambda_{1}\right)\right) / f_{g g}\left(g_{1}\right)$ and the dashed curve, $f_{g g}\left(g_{1}+g_{3}\left(\lambda_{2}\right)\right) / f_{g g}\left(g_{1}\right)$. The ratio of $f_{g g}\left(g_{1}\right)$ to $\exp \left(E^{\overline{\mathrm{MS}}}\right)$, given in the dot-dashed curve, compares the $g_{1}$ from Eq. (2.5) relative to the one-loop value in [2].

$$
\bar{F}_{g g, i}^{(0)}=\frac{\left[\left(s-s_{4}\right)^{2}-4 s m^{2}\right]^{1 / 2}}{2 s^{2}} F_{g g, i}^{B} .
$$

The differential with respect to $s_{4}$ of the two components yields

$$
\begin{aligned}
\frac{d \bar{F}_{g g, \mathrm{I}}^{(0)}\left(s, s_{4}, m^{2}\right)}{d s_{4}}= & -\frac{1}{2 s^{2} \sqrt{\left(s-s_{4}\right)^{2}-4 s m^{2}}}\left[2\left(s-s_{4}\right)\right. \\
& \left.-\frac{16 m^{2} s}{s-s_{4}}+\frac{320 m^{4} s^{2}}{\left(s-s_{4}\right)^{3}}-\frac{1024 m^{6} s^{3}}{\left(s-s_{4}\right)^{5}}\right]
\end{aligned}
$$



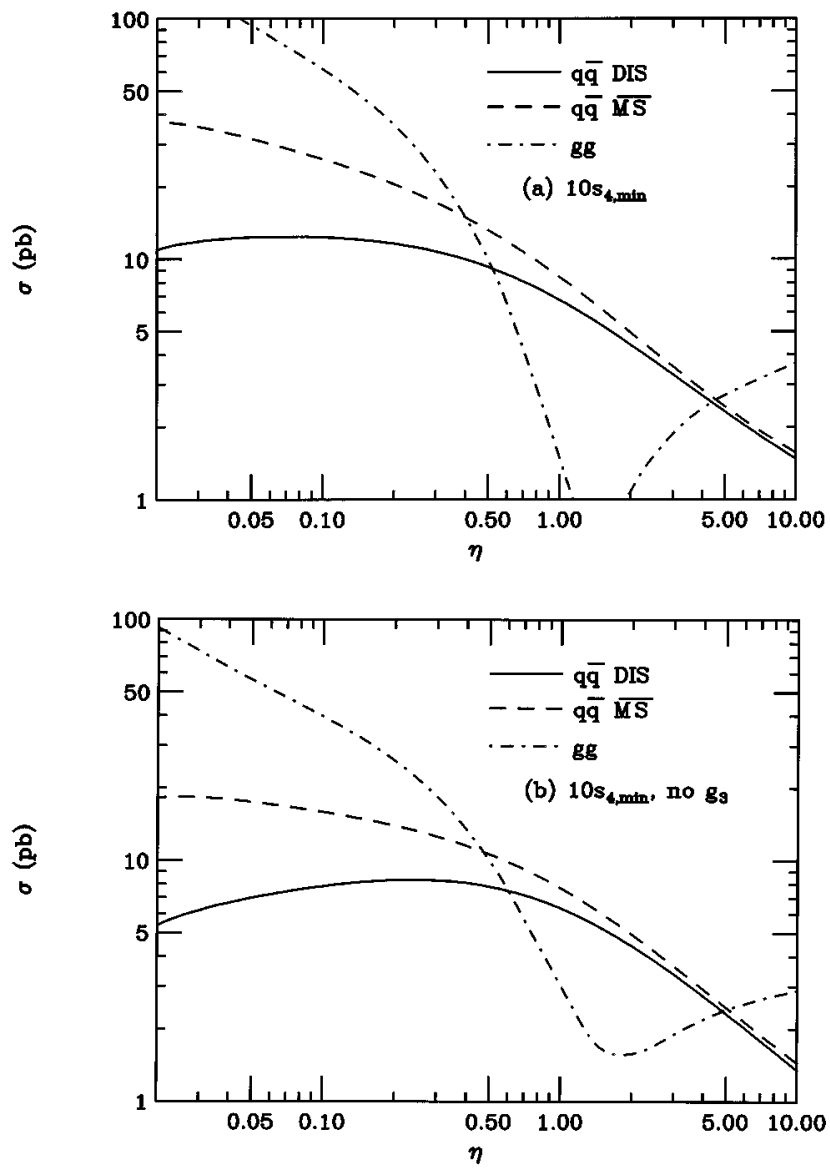

FIG. 4. The partonic top quark production cross section is shown as a function of $\eta=\left(s-4 m^{2}\right) /\left(4 m^{2}\right)$ for the $q \bar{q}$ channel in the DIS (solid) and $\overline{\mathrm{MS}}$ (dashed) schemes and the $g g$ (dot-dashed) channel. We use $s_{\text {cut }} \approx 10 s_{4 \text {,min }}$ with (a) and without (b) the NLL $g_{3}$ contributions, respectively.

$$
\begin{aligned}
\frac{d \bar{F}_{g g, I I}^{(0)}\left(s, s_{4}, m^{2}\right)}{d s_{4}} & \\
= & \frac{d \bar{F}_{g g, I}^{(0)}\left(s, s_{4}, m^{2}\right)}{d s_{4}}-\frac{1}{2 s^{2} \sqrt{\left(s-s_{4}\right)^{2}-4 s m^{2}}} \\
& \times\left[\frac{512 m^{6} s}{\left(s-s_{4}\right)^{3}}-\frac{6\left(s-s_{4}\right)^{3}}{s^{2}}-\frac{64 m^{4}}{s-s_{4}}\right] .
\end{aligned}
$$

Inserting the remaining color factors yields the $g g$ integrand in Eq. (3.2):

$$
\begin{aligned}
f_{g g}\left(\frac{s_{4}}{2 m^{2}}\right) \frac{d \bar{\sigma}_{g g}^{(0)}\left(s, s_{4}, m^{2}\right)}{d s_{4}} \\
=\frac{\pi}{8} K_{g g} N C_{F} \alpha_{s}^{2}\left\{\left[2 C_{F} f_{g g, 1}\left(\frac{s_{4}}{2 m^{2}}\right)+C_{F}\left(N^{2}-4\right)\right.\right. \\
\left.\left.\quad \times f_{g g, 2}\left(\frac{s_{4}}{2 m^{2}}\right)\right] \frac{d \bar{F}_{g g, \mathrm{I}}^{(0)}}{d s_{4}}+4 C_{A} f_{g g, 2}\left(\frac{s_{4}}{2 m^{2}}\right) \frac{d \bar{F}_{g g, \mathrm{II}}^{(0)}}{d s_{4}}\right\},
\end{aligned}
$$

where $K_{g g}=\left(N^{2}-1\right)^{-2}$ is a color average factor. One can numerically compare the coefficients of the color terms in
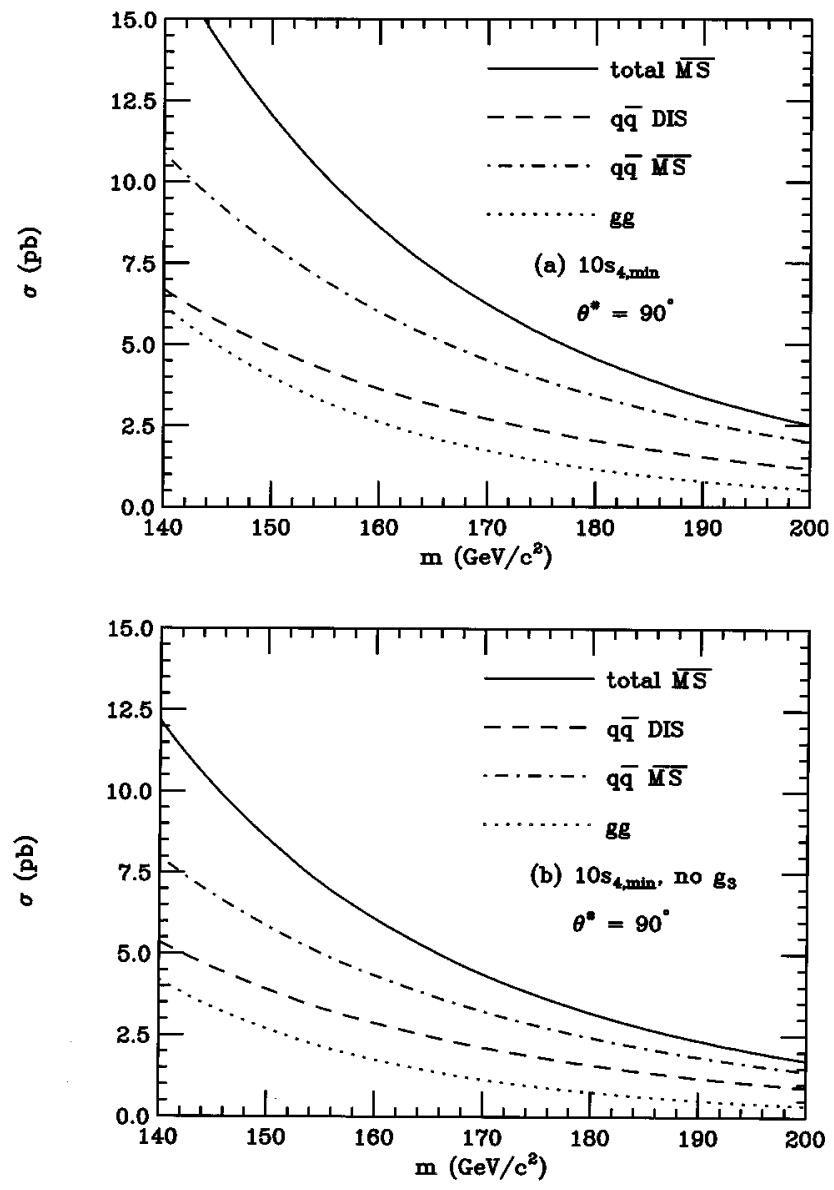

FIG. 5. The hadronic $t \bar{t}$ production cross section at $\theta^{*}=90^{\circ}$ is given as a function of top quark mass for $p \bar{p}$ collisions at the Tevatron energy $\sqrt{S}=1.8 \mathrm{TeV}$. We use the MRS $\mathrm{D}_{-}^{\prime}$ parton densities in the DIS scheme for $q \bar{q}$ annihilation (dashed) and in the $\overline{\mathrm{MS}}$ scheme for the $q \bar{q}$ (dot-dashed) and $g g$ (dotted) channels. The sum of the $q \bar{q}$ and $g g$ channels in the $\overline{\mathrm{MS}}$ scheme is given in the solid curve. The results are given for $s_{\text {cut }} \approx 10 s_{4 \text {,min }}$ with the NLL $g_{3}$ contributions in (a) and with these excluded in (b).

$d \sigma_{g g}^{(0)} / d s_{4}$ in Eq. (3.21) of Ref. [2] with the color terms near threshold in $d \bar{\sigma}_{g g}^{(0)} / d s_{4}$ in Eq. (3.19). Those in Ref. [2] are integrated over the angle $\theta^{*}$ before differentiation with respect to $s_{4}$ and are several times larger than those found here. Note that Eq. (3.19) contains several terms which can have different signs, making it a more complicated function of $s_{4}$. Therefore we do not expect our $g g$ results to agree with results obtained without attention to the color decomposition $[2,3,7,8]$.

Because the $\omega^{\prime}$ integral is cut off at $s_{4} /\left(2 m^{2}\right)$, the partonic cross section in Eq. (3.2) must also have a lower limit of $s_{\text {cut }}$ for the integrals to be finite. In earlier work up to LL $[2,3,7,8]$, the lower limit of the partonic cross section $s_{0}$ was taken to be $s_{0} / m^{2}=\left(\mu_{0} / \mu\right)^{2}$ in the DIS scheme and $s_{0} / m^{2}=\left(\mu_{0} / \mu\right)^{3}$ in the $\overline{\mathrm{MS}}$ scheme, where $\Lambda_{n_{\mathrm{f}}} \ll \mu_{0} \ll \mu$ and $\mu$ is the renormalization scale. It was assumed that the integral over the nonperturbative region below $s_{0}$ was negligible compared to the integral over the perturbative region. The final resummed cross section was obtained by comparison with the order-by-order approximate cross section up to $O\left(\alpha_{s}^{4}\right)$ as a function of $\mu_{0}$. Since each term in the perturba- 
tive result is positive, $\mu_{0}$ was chosen such that the resummed cross section was slightly larger than the sum of the approximate cross sections. It was found that for $\mu=m$ in the DIS scheme $\mu_{0} \approx(0.05-0.1) m$. Larger values were needed in the $\overline{\mathrm{MS}}$ scheme because of the cubic power of $\mu_{0} / \mu$. In the $q \bar{q}$ channel, $\mu_{0} \approx(0.1-0.2) m$, while $\mu_{0} \approx 0.35 m$ in the $g g$ channel. The $\mu_{0}$ needed in the $g g$ channel was larger because of the color factor $C_{A}$ in $E_{g g}$, see Eq. (3.14). As before, we will use the cutoff method since increasing $s_{\text {cut }}$ away from $s_{4, \min }$ reduces the scale and scheme dependence of the resummed perturbative cross section, as we discuss in detail in the next section and was also shown previously for the LL resummation [2,3]. In the next section, we will calculate the $\mu_{0}$ appropriate for our chosen $s_{\text {cut }}$.

\section{NUMERICAL RESULTS}

We first present our results for top quark production. In Fig. 1(a) we plot the exponents contributing to $f_{q \bar{q}}^{\mathrm{DIS}}$ in Eq. (3.3) at $\theta^{*}=90^{\circ}$ as a function of $s_{4} / 2 m^{2}$ with $m=175 \mathrm{GeV} /$ $c^{2}$ and $\sqrt{s}=351 \mathrm{GeV}$. Since only the octet component contributes to $g_{3}$ here, only $E_{q q}-\left(\lambda_{2}\right)$ is shown. We also show, for comparison, the one-loop calculation of $g_{1}, E^{\text {DIS }}$, given in [2]. The one-loop and two-loop results are quite similar until $s_{4}$ approaches $s_{4, \min }$. As $\omega^{\prime} Q / \Lambda \rightarrow 1, E_{q \bar{q}}^{\mathrm{DIS}}\left(g_{1}\right)$ is larger since $E^{\text {DIS }}$ was smoothed near the cutoff [2]. The sum $E_{q \bar{q}}^{\mathrm{DIS}}+E_{q \bar{q}}\left(\lambda_{2}\right)$ is always larger than $E^{\mathrm{DIS}}$ because the $g_{3}$ contribution compensates for the small, negative $g_{2}$ component $\left(\left|E_{q \bar{q}}^{\mathrm{DIS}}\left(g_{2}\right)\right|\right.$ is shown). The power of $1-z$ in $\alpha_{s}$ determines the slope of the exponents at small $s_{4} / 2 \mathrm{~m}^{2}$. The $g_{2}$ contribution, linear in $1-z$, is the flattest as $s_{4} / 2 m^{2} \rightarrow 0$. The $g_{1}$ component shows more growth, diverging from $E^{\text {DIS }}$ near $s_{4, \text { min }}$, and the $g_{3}$ contribution, quadratic in $1-z$, grows fastest. The growth of the sum is intermediate to that of $E_{q \bar{q}}^{\mathrm{DIS}}\left(g_{1}\right)$ and $E_{q} \bar{q}\left(\lambda_{2}\right)$.

To better illustrate the importance of these contributions to the partonic cross section, we show the relative enhancements as a function of $s_{4} / 2 \mathrm{~m}^{2}$ in Fig. 1(b). The negative contribution and the slow growth of $g_{2}$ near $s_{4, \min }$ are reflected in the near threshold behavior of $f_{q} \bar{q}\left(g_{1}+g_{2}\right) /$ $f_{q} \bar{q}\left(g_{1}\right)$, where, e.g., $f_{q \bar{q}}-\left(g_{1}\right) \equiv \exp \left[E_{q \bar{q}}^{\text {DIS }}\left(g_{1}\right)\right]$. In the intermediate range of $s_{4} / 2 m^{2}, E^{\mathrm{DIS}}$ is larger than $E_{q}^{\mathrm{DIS}}\left(g_{1}\right)$. Although $E_{q}-\left(\lambda_{2}\right)$ dominates $f_{q \bar{q}}^{\mathrm{DIS}}$ as $s_{4} / 2 m^{2} \rightarrow 1$, the enhancement in this region is not very significant. Thus the choice of the cutoff determines the importance of the NLL contributions. However, a large NLL correction is not necessarily reflected in the hadronic cross section since it is the parton luminosity relative to the equivalent $\eta=s / 4 m^{2}-1$ that determines the true strength of the corrections.

Similar results are seen for the exponents in the $\overline{\mathrm{MS}}$ scheme, shown in Fig. 2. The $g_{2}$ term vanishes in this scheme. The change in the upper limit of the $\xi$ integral increases the phase space of $E_{q \bar{q}}^{\overline{\mathrm{MS}}}$. Both these changes enhance the $\overline{\mathrm{MS}}$ result. The $g_{3}$ contribution is identical to that shown in Fig. 1.

In Fig. 3 we plot the corresponding results for the $g g$ channel in the $\overline{\mathrm{MS}}$ scheme. Note that the real parts of the $\lambda_{i}$ 's are the same in Eqs. (2.11) and (2.13) so that the strength of the $g_{3}$ contributions are the same in the $q \bar{q}$ and $g g$ channels. However, since different color structures in the $g g$ channel multiply different derivatives of the Born cross section, there is no single sum involving the NLL components as in the $q \bar{q}$ channel. The $E_{g g}\left(\lambda_{1}\right)$ component is quite small and negative near threshold, making the sum $E_{g g}+E_{g g}\left(\lambda_{1}\right)$ indistinguishable from $E_{g g}$. The $E_{g g}\left(\lambda_{2}\right)$ contribution is the same as in the $q \bar{q}$ channel, resulting in the larger sum $E_{g g}+E_{g g}\left(\lambda_{2}\right)$ shown in the dot-dashed curve.

Note that changing the scale effectively shifts the range of $s_{4} / 2 \mathrm{~m}^{2}$. We found also that, for the same value of $s_{4} / 2 \mathrm{~m}^{2}$, the exponents are larger for smaller values of $Q^{2}$, as expected.

We have checked the energy dependence of the $g_{3}$ contributions. We find that $E_{g g}\left(\lambda_{1}\right)$ is always negative, decreasing the NLL correction for all $s$. Its energy dependence is quite strong and thus above threshold it serves to damp the resummation. On the other hand, there is very little energy dependence of $E_{I J}\left(\lambda_{2}\right)$ close to threshold. As $s$ grows large, the $\ln \left(m^{2} s / t_{1}^{2}\right)$ term in Eqs. (2.11) and (2.13) dominates the energy dependence, causing $E_{I J}\left(\lambda_{2}\right)$ to change sign, eventually leading to a strong damping of the NLL component (and probably leading to a smooth energy variation of the resummed cross section above threshold). This logarithmic term also has the only explicit $s_{4}^{\prime}$ dependence of the $g_{3}$ contribution at $\theta^{*}=90^{\circ}$ apart from the argument of $\alpha_{s}$. Since $\lambda_{1}$ only depends on $s_{4}^{\prime}$ through $\alpha_{s}$, these contributions have similar behaviors in $s$.

In Fig. 4 we plot the partonic $t \bar{t}$ cross section as a function of $\eta$ for the $q \bar{q}$ and $g g$ channels. At $\eta \approx 1$ the $g g$ contribution is numerically smaller than the corresponding $q \bar{q}$ result. The importance of the NLL contributions to the partonic cross section depends on the lower limit of the $s_{4}$ integral. We have chosen $s_{\text {cut }}=10 s_{4, \min }$, corresponding to $\mu_{0}=0.26 \mathrm{~m}$ in the $\overline{\mathrm{MS}}$ scheme and $0.13 \mathrm{~m}$ in the DIS scheme when $Q^{2}=m^{2}$, similar to the values used previously. Changing $s_{\text {cut }}$ changes the effective $\eta$ range. However, note that for $\eta \geqslant 1$, the $q \bar{q}$ results are nearly scheme independent. The $q \bar{q}$ result in the $\overline{\mathrm{MS}}$ scheme is always larger than the DIS result. We have checked that the dependence on $s_{\text {cut }}$ is rather weak in this channel. The $g g$ channel is similar although the different color structure of the NLL terms leads to a stronger variation over all $\eta$ in this channel.

To illustrate the contribution of the NLL terms to the partonic cross section, in Fig. 4(b) we show the partonic cross section with only the LL contributions for the same value of $s_{\text {cut }}$. At $\eta>1$ the $q \bar{q}$ contributions with and without the NLL components are nearly the same but at $\eta \leqslant 0.02$ the $g_{3}$ contribution makes the partonic cross section up to a factor of 2 larger. Both with and without the NLL terms, the scheme dependence increases as $\eta$ decreases.

We note that for fixed angle $\theta^{*}=90^{\circ}$, the partonic cross section in the $g g$ channel can become negative around $\eta \sim 1-2$. Because $d \bar{F}_{g g, \mathrm{II}}^{(0)} / d s_{4}$ is generally opposite in sign to $d \bar{F}_{g g, \mathrm{I}}^{(0)} / d s_{4}$, when it grows larger than $d \bar{F}_{g g, \mathrm{I}}^{(0)} / d s_{4}$ the partonic $g g$ cross section becomes negative. In our calculation of the hadronic cross section, we exclude the negative region 
TABLE I. The hadronic $t \bar{t}$ production cross sections in $p \bar{p}$ collisions at $\sqrt{S}=1.8 \mathrm{TeV}$ for $s_{\text {cut }}=10 s_{4, \min }$ and $\theta^{*}=90^{\circ}$. The effective $\mu_{0}$ from [2] is given for each $Q^{2}$.

\begin{tabular}{|c|c|c|c|c|c|c|}
\hline$m\left(\mathrm{GeV} / c^{2}\right)$ & $\sigma_{q \bar{q}}^{\mathrm{DIS}}(\mathrm{pb})$ & $\begin{array}{c}\sigma_{q \bar{q}}^{\mathrm{NLL}}(\mathrm{pb}) \\
\overline{\mathrm{MS}}\end{array}$ & $\sigma_{g g}(\mathrm{pb})$ & $\sigma_{q \bar{q}}^{\mathrm{DIS}}(\mathrm{pb})$ & $\sigma_{q \bar{q}}^{\mathrm{LL}}(\mathrm{pb})$ & $\sigma_{g g}(\mathrm{pb})$ \\
\hline & \multicolumn{6}{|c|}{$Q^{2}=m^{2} \Rightarrow \mu_{0}^{\mathrm{DIS}}=0.13 m, \mu_{0}^{\overline{\mathrm{MS}}}=0.26 m$} \\
\hline 150 & 4.91 & 8.04 & 3.99 & 3.91 & 5.85 & 2.68 \\
\hline 155 & 4.21 & 6.94 & 3.21 & 3.32 & 5.01 & 2.15 \\
\hline 160 & 3.63 & 5.99 & 2.61 & 2.87 & 4.33 & 1.75 \\
\hline 165 & 3.13 & 5.20 & 2.12 & 2.47 & 3.74 & 1.40 \\
\hline 170 & 2.71 & 4.51 & 1.73 & 2.12 & 3.23 & 1.14 \\
\hline 175 & 2.34 & 3.92 & 1.41 & 1.84 & 2.80 & 0.93 \\
\hline 180 & 2.03 & 3.41 & 1.15 & 1.59 & 2.43 & 0.75 \\
\hline 185 & 1.77 & 2.98 & 0.94 & 1.37 & 2.11 & 0.62 \\
\hline 190 & 1.53 & 2.59 & 0.77 & 1.19 & 1.84 & 0.50 \\
\hline 195 & 1.33 & 2.27 & 0.63 & 1.03 & 1.60 & 0.41 \\
\hline \multirow[t]{2}{*}{200} & 1.16 & 1.99 & 0.52 & 0.90 & 1.39 & 0.34 \\
\hline & \multicolumn{6}{|c|}{$Q^{2}=4 m^{2} \Rightarrow \mu_{0}^{\mathrm{DIS}}=0.19 m, \mu_{0}^{\overline{\mathrm{MS}}}=0.41 m$} \\
\hline 150 & 3.83 & 6.60 & 4.94 & 3.06 & 4.74 & 3.11 \\
\hline 155 & 3.30 & 5.71 & 3.95 & 2.61 & 4.07 & 2.49 \\
\hline 160 & 2.83 & 4.92 & 3.22 & 2.24 & 3.50 & 2.01 \\
\hline 165 & 2.44 & 4.27 & 2.61 & 1.93 & 3.03 & 1.62 \\
\hline 170 & 2.10 & 3.69 & 2.13 & 1.65 & 2.61 & 1.32 \\
\hline 175 & 1.82 & 3.21 & 1.74 & 1.43 & 2.26 & 1.08 \\
\hline 180 & 1.56 & 2.78 & 1.42 & 1.23 & 1.95 & 0.88 \\
\hline 185 & 1.36 & 2.42 & 1.16 & 1.06 & 1.69 & 0.71 \\
\hline 190 & 1.18 & 2.11 & 0.96 & 0.92 & 1.47 & 0.59 \\
\hline 195 & 1.03 & 1.84 & 0.79 & 0.80 & 1.28 & 0.48 \\
\hline \multirow[t]{2}{*}{200} & 0.89 & 1.61 & 0.65 & 0.69 & 1.11 & 0.39 \\
\hline & \multicolumn{6}{|c|}{$Q^{2}=m^{2} / 4 \Rightarrow \mu_{0}^{\mathrm{DIS}}=0.094 m, \mu_{0}^{\overline{\mathrm{MS}}}=0.16 m$} \\
\hline 150 & 6.20 & 9.53 & 3.51 & 4.97 & 7.13 & 2.51 \\
\hline 155 & 5.36 & 8.28 & 2.84 & 4.26 & 6.12 & 2.03 \\
\hline 160 & 4.62 & 7.17 & 2.29 & 3.67 & 5.29 & 1.62 \\
\hline 165 & 4.00 & 6.24 & 1.85 & 3.16 & 4.57 & 1.31 \\
\hline 170 & 3.46 & 5.41 & 1.51 & 2.73 & 3.97 & 1.06 \\
\hline 175 & 3.00 & 4.72 & 1.23 & 2.36 & 3.44 & 0.86 \\
\hline 180 & 2.61 & 4.11 & 1.00 & 2.04 & 2.98 & 0.70 \\
\hline 185 & 2.27 & 3.59 & 0.82 & 1.77 & 2.60 & 0.57 \\
\hline 190 & 1.99 & 3.16 & 0.68 & 1.54 & 2.26 & 0.47 \\
\hline 195 & 1.73 & 2.75 & 0.55 & 1.34 & 1.97 & 0.38 \\
\hline 200 & 1.51 & 2.42 & 0.46 & 1.16 & 1.72 & 0.31 \\
\hline
\end{tabular}

if it occurs. For the particular values of $s_{\text {cut }}$ and $Q^{2}$ shown here, the partonic cross section is always positive. However, for other values of $s_{\text {cut }}$ and $Q^{2}$ the change in sign can occur, both with and without the $g_{3}$ contribution and thus is not just a feature of the NLL contribution at this angle.

The results in Fig. 4 imply that the relative importance of the NLL contributions to the hadronic cross section depend on which regions in $\eta$ are weighted most heavily by the convolution with the parton densities. With equivalent parton luminosities, if $\eta \approx 0.5$, then we expect the $g g$ contribution to be comparable to that of the $q \bar{q}$ channel while if $\eta \approx 2$ then we expect the $q \bar{q}$ channel to dominate. Thus the parton luminosity in the $x$ space probed by the hadronic cross section determines the ultimate importance of the NLL contri- bution. A comparison with previous results obtained by integrating over the angle $\theta^{*}[2,3]$ is inconclusive since here we work at fixed $\theta^{*}$. Finally we note that previously $[2,3,7,8]$ different cutoffs were chosen in the $q \bar{q}$ and $g g$ channels to match the order-by-order contributions, as described at the end of the previous section, whereas here we keep the same $s_{\text {cut }}$ for both channels.

The hadronic cross section calculated to NLL is

$$
\begin{aligned}
\sigma^{\mathrm{NLL}}\left(S, m^{2}\right)= & \sum_{i j} \int_{\tau_{0}}^{1} d \tau \int_{\tau}^{1} \frac{d x}{x} f_{i}^{h_{1}}\left(x, \mu^{2}\right) \\
& \times f_{j}^{h_{2}}\left(\frac{\tau}{x}, \mu^{2}\right) \sigma_{i j}\left(\tau S, m^{2}\right),
\end{aligned}
$$


TABLE II. The hadronic $t \bar{t}$ production cross sections in $p \bar{p}$ collisions at $\sqrt{S}=1.8 \mathrm{TeV}$ for $s_{\text {cut }}=5 s_{4, \min }$ and $\theta^{*}=90^{\circ}$. The effective $\mu_{0}$ from [2] is given for each $Q^{2}$.

\begin{tabular}{|c|c|c|c|c|c|c|}
\hline \multirow[t]{2}{*}{$m\left(\mathrm{GeV} / c^{2}\right)$} & $\sigma_{q \bar{q}}^{\mathrm{DIS}}(\mathrm{pb})$ & $\begin{array}{c}\mathrm{NLL} \\
\sigma_{q \bar{q}}^{\overline{\mathrm{MS}}}(\mathrm{pb})\end{array}$ & $\sigma_{g g}(\mathrm{pb})$ & $\sigma_{q \bar{q}}^{\mathrm{DIS}}(\mathrm{pb})$ & $\sigma_{q \bar{q}}^{\frac{\mathrm{LL}}{\mathrm{MS}}}(\mathrm{pb})$ & $\sigma_{g g}(\mathrm{pb})$ \\
\hline & \multicolumn{6}{|c|}{$Q^{2}=m^{2} \Rightarrow \mu_{0}^{\mathrm{DIS}}=0.094 m, \mu_{0}^{\overline{\mathrm{MS}}}=0.21 m$} \\
\hline 150 & 5.43 & 10.81 & 17.31 & 4.13 & 7.07 & 9.67 \\
\hline 155 & 4.65 & 9.29 & 14.13 & 3.55 & 6.09 & 7.89 \\
\hline 160 & 4.01 & 8.08 & 11.66 & 3.05 & 5.25 & 6.44 \\
\hline 165 & 3.47 & 7.02 & 9.54 & 2.63 & 4.55 & 5.27 \\
\hline 170 & 2.99 & 6.10 & 7.93 & 2.25 & 3.93 & 4.30 \\
\hline 175 & 2.59 & 5.32 & 6.53 & 1.95 & 3.41 & 3.54 \\
\hline 180 & 2.25 & 4.63 & 5.38 & 1.69 & 2.96 & 2.90 \\
\hline 185 & 1.95 & 4.04 & 4.47 & 1.46 & 2.58 & 2.41 \\
\hline 190 & 1.70 & 3.56 & 3.68 & 1.26 & 2.24 & 1.99 \\
\hline 195 & 1.48 & 3.09 & 3.06 & 1.10 & 1.96 & 1.65 \\
\hline \multirow[t]{2}{*}{200} & 1.28 & 2.71 & 2.55 & 0.96 & 1.71 & 1.37 \\
\hline & \multicolumn{6}{|c|}{$Q^{2}=4 m^{2} \Rightarrow \mu_{0}^{\mathrm{DIS}}=0.13 m, \mu_{0}^{\overline{\mathrm{MS}}}=0.33 m$} \\
\hline 150 & 4.15 & 8.80 & 26.50 & 3.20 & 5.68 & 14.01 \\
\hline 155 & 3.55 & 7.58 & 21.69 & 2.73 & 4.86 & 11.32 \\
\hline 160 & 3.06 & 6.55 & 17.85 & 2.34 & 4.19 & 9.22 \\
\hline 165 & 2.63 & 5.68 & 14.66 & 2.01 & 3.63 & 7.57 \\
\hline 170 & 2.28 & 4.95 & 12.03 & 1.73 & 3.12 & 6.26 \\
\hline 175 & 1.96 & 4.29 & 10.03 & 1.49 & 2.71 & 5.13 \\
\hline 180 & 1.70 & 3.74 & 8.24 & 1.28 & 2.34 & 4.23 \\
\hline 185 & 1.47 & 3.26 & 6.86 & 1.11 & 2.04 & 3.51 \\
\hline 190 & 1.28 & 2.85 & 5.65 & 0.96 & 1.77 & 2.88 \\
\hline 195 & 1.11 & 2.48 & 4.74 & 0.83 & 1.54 & 2.38 \\
\hline \multirow[t]{2}{*}{200} & 0.96 & 2.17 & 3.89 & 0.72 & 1.34 & 1.98 \\
\hline & \multicolumn{6}{|c|}{$Q^{2}=m^{2} / 4 \Rightarrow \mu_{0}^{\mathrm{DIS}}=0.067 m, \mu_{0}^{\overline{\mathrm{MS}}}=0.13 m$} \\
\hline 150 & 7.10 & 13.16 & 12.44 & 5.41 & 8.84 & 7.42 \\
\hline 155 & 6.13 & 11.44 & 10.21 & 4.65 & 7.63 & 6.04 \\
\hline 160 & 5.30 & 9.91 & 8.35 & 3.99 & 6.58 & 4.91 \\
\hline 165 & 4.58 & 8.65 & 6.86 & 3.44 & 5.69 & 4.03 \\
\hline 170 & 3.95 & 7.49 & 5.64 & 2.97 & 4.94 & 3.28 \\
\hline 175 & 3.44 & 6.57 & 4.65 & 2.57 & 4.29 & 2.71 \\
\hline 180 & 2.98 & 5.72 & 3.84 & 2.23 & 3.75 & 2.25 \\
\hline 185 & 2.60 & 5.02 & 3.20 & 1.93 & 3.25 & 1.84 \\
\hline 190 & 2.27 & 4.40 & 2.65 & 1.68 & 2.84 & 1.52 \\
\hline 195 & 1.98 & 3.85 & 2.19 & 1.46 & 2.48 & 1.25 \\
\hline 200 & 1.73 & 3.38 & 1.82 & 1.28 & 2.17 & 1.04 \\
\hline
\end{tabular}

where $\sigma_{i j}\left(\tau S, m^{2}\right)$ is the partonic cross section, Eq. (3.2), and $\tau_{0}=\left(m+\sqrt{m^{2}+s_{\text {cut }}}\right)^{2} / S$. We evaluate the parton densities at $\mu^{2}=Q^{2}$. Since the parton densities are only available at fixed order, the application to a resummed cross section introduces some uncertainty. We have used the MartinRoberts-Stirling set $\mathrm{D}_{-}^{\prime}$ (MRS $\mathrm{D}_{-}^{\prime}$ ) DIS densities for the $q \bar{q}$ DIS channel and the MRS $\mathrm{D}_{-}^{\prime} \overline{\mathrm{MS}}$ densities $[18,19]$ for both channels in this scheme. ${ }^{1}$ Note that $\Lambda_{5}=0.1559 \mathrm{GeV}$ for both sets. The result is not strongly dependent on the parton distributions in the $x$ range probed at this energy,

\footnotetext{
${ }^{1}$ The $x$ dependence of the parton densities in the two schemes is very similar.
}

$x \sim 0.2$ at $y=0$ for $m=175 \mathrm{GeV} / c^{2}$, entering primarily through the value of $\Lambda_{5}$ since different sets of parton densities have a different $\Lambda_{5}$, changing $s_{4, \min }$ as well as the value of $\alpha_{s}$. Taking $\alpha_{s}$ to only one loop, as in [2], increases $\alpha_{s}$ and thus the hadronic cross section. For a more complete discussion of the parton density and $\alpha_{s}$ dependence, see [8].

In Fig. 5(a) we plot the $t \bar{t}$ production cross section at $\theta^{*}=90^{\circ}$ as a function of top quark mass at the Fermilab Tevatron with $\sqrt{S}=1.8 \mathrm{TeV}$ in the DIS and $\overline{\mathrm{MS}}$ schemes for the $q \bar{q}$ channel and the $\overline{\mathrm{MS}}$ scheme for the $g g$ channel with $s_{\text {cut }}=10 s_{4, \min }$ and $Q^{2}=m^{2}$. The total $\overline{\mathrm{MS}}$ cross section at $\theta^{*}=90^{\circ}$ is $5.3 \mathrm{pb}$ at $m=175 \mathrm{GeV} / \mathrm{c}^{2}$, similar to the angleintegrated results of Ref. [5]. Previous NLO results showed that for $m=100 \mathrm{GeV} / c^{2}$, even though $\eta_{\max }=80$ the total 
TABLE III. The hadronic $t \bar{t}$ production cross sections in $p \bar{p}$ collisions at $\sqrt{S}=1.8 \mathrm{TeV}$ for $s_{\text {cut }}=20 s_{4, \min }$ and $\theta^{*}=90^{\circ}$. The effective $\mu_{0}$ from [2] is given for each $Q^{2}$.

\begin{tabular}{|c|c|c|c|c|c|c|}
\hline \multirow[t]{2}{*}{$m\left(\mathrm{GeV} / c^{2}\right)$} & $\sigma_{q \bar{q}}^{\mathrm{DIS}}(\mathrm{pb})$ & $\frac{\mathrm{NLL}}{\sigma_{q \overline{\mathrm{MS}}}^{\mathrm{MS}}(\mathrm{pb})}$ & $\sigma_{g g}(\mathrm{pb})$ & $\sigma_{q \bar{q}}^{\mathrm{DIS}}(\mathrm{pb})$ & $\frac{\mathrm{LL}}{\sigma_{q \bar{q}}^{\overline{\mathrm{MS}}}(\mathrm{pb})}$ & $\sigma_{g g}(\mathrm{pb})$ \\
\hline & \multicolumn{6}{|c|}{$Q^{2}=m^{2} \Rightarrow \mu_{0}^{\mathrm{DIS}}=0.19 m, \mu_{0}^{\overline{\mathrm{MS}}}=0.33 m$} \\
\hline 150 & 4.40 & 6.30 & 1.61 & 3.61 & 4.90 & 1.24 \\
\hline 155 & 3.78 & 5.43 & 1.29 & 3.10 & 4.23 & 0.98 \\
\hline 160 & 3.25 & 4.69 & 1.03 & 2.66 & 3.64 & 0.78 \\
\hline 165 & 2.80 & 4.06 & 0.83 & 2.28 & 3.13 & 0.63 \\
\hline 170 & 2.42 & 3.53 & 0.67 & 1.97 & 2.71 & 0.50 \\
\hline 175 & 2.10 & 3.06 & 0.54 & 1.70 & 2.34 & 0.40 \\
\hline 180 & 1.82 & 2.67 & 0.44 & 1.47 & 2.03 & 0.33 \\
\hline 185 & 1.58 & 2.32 & 0.35 & 1.27 & 1.76 & 0.26 \\
\hline 190 & 1.37 & 2.02 & 0.29 & 1.10 & 1.53 & 0.21 \\
\hline 195 & 1.19 & 1.76 & 0.23 & 0.96 & 1.33 & 0.17 \\
\hline \multirow[t]{2}{*}{200} & 1.04 & 1.55 & 0.19 & 0.83 & 1.16 & 0.14 \\
\hline & \multicolumn{6}{|c|}{$Q^{2}=4 m^{2} \Rightarrow \mu_{0}^{\mathrm{DIS}}=0.27 m, \mu_{0}^{\overline{\mathrm{MS}}}=0.52 m$} \\
\hline 150 & 3.55 & 5.36 & 1.70 & 2.91 & 4.11 & 1.24 \\
\hline 155 & 3.05 & 4.61 & 1.36 & 2.48 & 3.52 & 0.99 \\
\hline 160 & 2.62 & 3.98 & 1.09 & 2.13 & 3.03 & 0.79 \\
\hline 165 & 2.25 & 3.44 & 0.87 & 1.83 & 2.61 & 0.63 \\
\hline 170 & 1.94 & 2.97 & 0.71 & 1.57 & 2.25 & 0.51 \\
\hline 175 & 1.68 & 2.58 & 0.57 & 1.35 & 1.94 & 0.41 \\
\hline 180 & 1.45 & 2.24 & 0.46 & 1.17 & 1.68 & 0.33 \\
\hline 185 & 1.26 & 1.95 & 0.38 & 1.01 & 1.46 & 0.27 \\
\hline 190 & 1.09 & 1.69 & 0.31 & 0.87 & 1.26 & 0.22 \\
\hline 195 & 0.95 & 1.48 & 0.25 & 0.75 & 1.09 & 0.18 \\
\hline \multirow[t]{2}{*}{200} & 0.82 & 1.28 & 0.20 & 0.69 & 0.96 & 0.14 \\
\hline & \multicolumn{6}{|c|}{$Q^{2}=m^{2} / 4 \Rightarrow \mu_{0}^{\mathrm{DIS}}=0.13 m, \mu_{0}^{\overline{\mathrm{MS}}}=0.21 m$} \\
\hline 150 & 5.27 & 7.10 & 1.57 & 4.40 & 5.68 & 1.25 \\
\hline 155 & 4.54 & 6.14 & 1.25 & 3.77 & 4.89 & 1.00 \\
\hline 160 & 3.92 & 5.31 & 0.99 & 3.25 & 4.23 & 0.80 \\
\hline 165 & 3.39 & 4.62 & 0.80 & 2.80 & 3.65 & 0.64 \\
\hline 170 & 2.94 & 4.02 & 0.64 & 2.41 & 3.16 & 0.51 \\
\hline 175 & 2.55 & 3.49 & 0.52 & 2.08 & 2.74 & 0.41 \\
\hline 180 & 2.22 & 3.05 & 0.42 & 1.81 & 2.38 & 0.33 \\
\hline 185 & 1.93 & 2.67 & 0.34 & 1.57 & 2.07 & 0.27 \\
\hline 190 & 1.68 & 2.33 & 0.28 & 1.37 & 1.80 & 0.22 \\
\hline 195 & 1.47 & 2.04 & 0.23 & 1.18 & 1.57 & 0.18 \\
\hline 200 & 1.28 & 1.78 & 0.18 & 1.03 & 1.37 & 0.14 \\
\hline
\end{tabular}

hadronic cross section was obtained already for $\eta \approx 3$ (cf. Fig. 1 in [3]). When $m=175 \mathrm{GeV} / c^{2}, \eta_{\max }=26$. Comparing Figs. 4(a) and 5(a), we can infer that in this case the important $\eta$ region is $\eta \approx 0.3$. At this $\eta$ the $q \bar{q}$ luminosity is six times larger than the $g g$ luminosity.

To quantify the enhancement in the $t \bar{t}$ cross section at fixed angle due to the NLL terms, in Fig. 5(b) we show our results with the same values of $s_{\text {cut }}$ and $Q^{2}$ but without the NLL contributions, i.e., no $g_{3}$ component. The NLL contribution increases with top mass at this energy. The DIS cross section is enhanced between 25 and $30 \%$ as the top mass increases from 140 to $200 \mathrm{GeV} / c^{2}$. The enhancement is somewhat larger for the $\overline{\mathrm{MS}}$ scheme, between 36 and $43 \%$ in the $q \bar{q}$ channel and 47 to $55 \%$ in the $g g$ channel. The change in the amount of enhancement is, in part, due to the energy dependence of the $g_{3}$ contribution. For our choice of $s_{\text {cut }}$, the $t \bar{t}$ cross section at $m=175 \mathrm{GeV} / c^{2}$ without the NLL $g_{3}$ contribution is $3.7 \mathrm{pb}$. Thus the NLL terms enhance the total $\overline{\mathrm{MS}}$ cross section at $\theta^{*}=90^{\circ}$ by $43 \%$ at this mass and value of $s_{\text {cut }}$ in our calculation. The relatively large contribution from the NLL terms verifies that the lower $\eta$ region is most important.

We have further investigated the $s_{\text {cut }}$ and scale dependence of our calculation. In Tables I-III we give the numerical values of the hadronic top quark production cross sections at LL and NLL with $s_{\text {cut }}=10 s_{4, \min }, 5 s_{4, \min }$, and $20 s_{4, \min }$, respectively. For each value of $s_{\text {cut }}$ we show the results for $Q^{2}=m^{2}, 4 m^{2}$, and $m^{2} / 4$. In the tables we have 

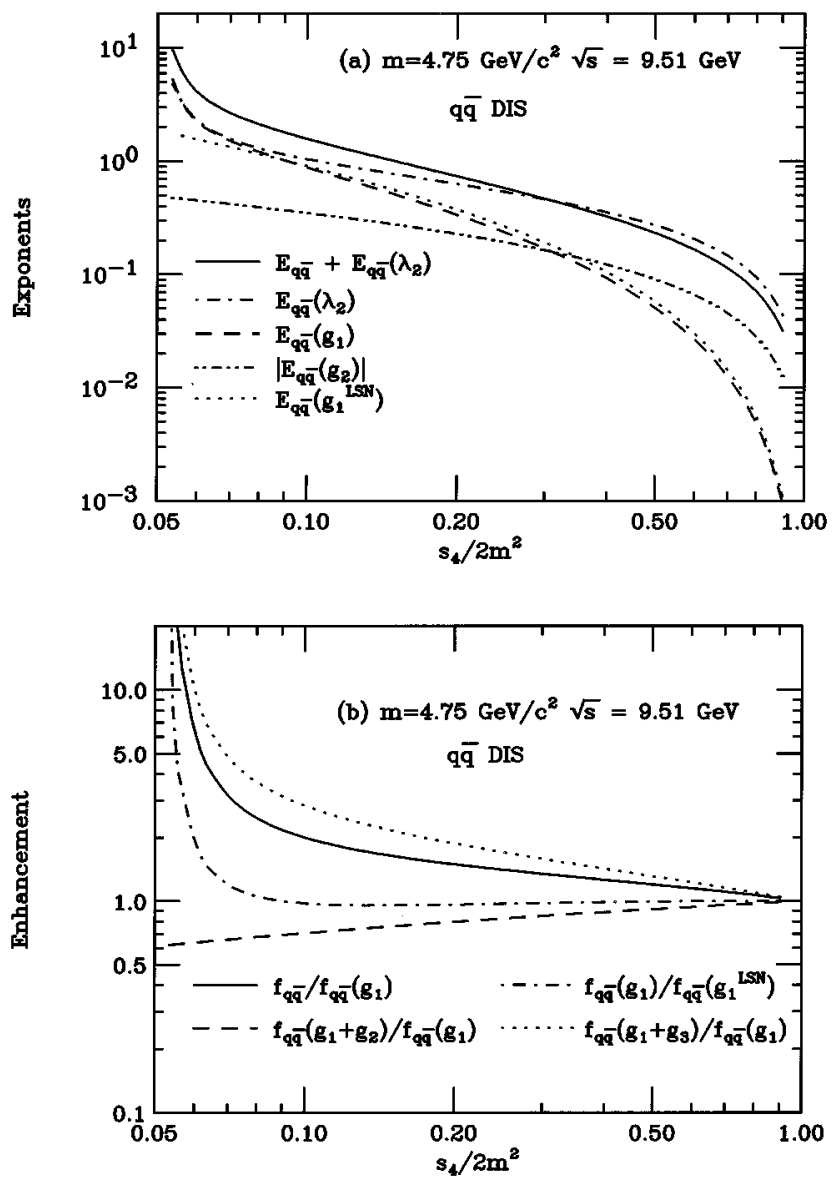

FIG. 6. We show the contributions to $f_{q}^{\mathrm{DIS}}$ and the enhancement factors in bottom quark production for $m=4.75 \mathrm{GeV} / c^{2}$ and $\sqrt{s}=9.51 \mathrm{GeV}$. The labels are as in Fig. 1 .

also indicated the equivalent value of the cutoff $\mu_{0}$ defined in [2] in both the DIS and $\overline{\mathrm{MS}}$ schemes. Note that in Tables I and III, the scale dependence of the $g g$ channel is weak compared to the $q \bar{q}$ contributions. When $s_{\text {cut }}=10 s_{4, \min }$, the $g g$ cross section changes $41 \%$ between $Q^{2}=m^{2} / 4$ and $4 m^{2}$ at $m=175 \mathrm{GeV} / c^{2}$. In contrast, the $q \bar{q} \overline{\mathrm{MS}}$ cross section changes $47 \%$ and the DIS cross section by $65 \%$. At the same mass but with $s_{\text {cut }}=20 s_{4 \text {, min }}$, the $g g$ cross section changes $10 \%$ between the highest and lowest scales while the $q \bar{q}$ $\overline{\mathrm{MS}}$ cross section changes $35 \%$ and the DIS cross section $52 \%$. On the other hand, for the lowest $s_{\text {cut }}$ the $g g$ cross section has the largest scale dependence, more than a factor of 2 change while the $\overline{\mathrm{MS}}$ cross section changes $53 \%$ in the $q \bar{q}$ channel.

Note that for a fixed value of $s_{\text {cut }}$, the $g g$ cross section is actually largest when $Q^{2}=4 \mathrm{~m}^{2}$ than at lower $Q^{2}$. This result is counterintuitive since one generally expects that increasing the scale decreases the cross section. This is true in the $q \bar{q}$ channel and also in the $g g$ channel when $\eta>1$. At fixed $s_{4}$, the exponents are larger for lower values of the scale due to the running of $\alpha_{s}$. However, the difference in the factors $f_{g g, 1}$ and $f_{g g, 2}$ at low $\eta$ shifts the relative weights of $d \bar{F}_{g g, \mathrm{I}}^{(0)} / d s_{4}$ and $d \bar{F}_{g g, \mathrm{II}}^{(0)} / d s_{4}$ so that the partonic cross section is largest for the largest scale studied $Q^{2}=4 \mathrm{~m}^{2}$, when $\eta<0.5$. In addition, changing the scale for fixed $s_{\text {cut }} / s_{4, \text { min }}$
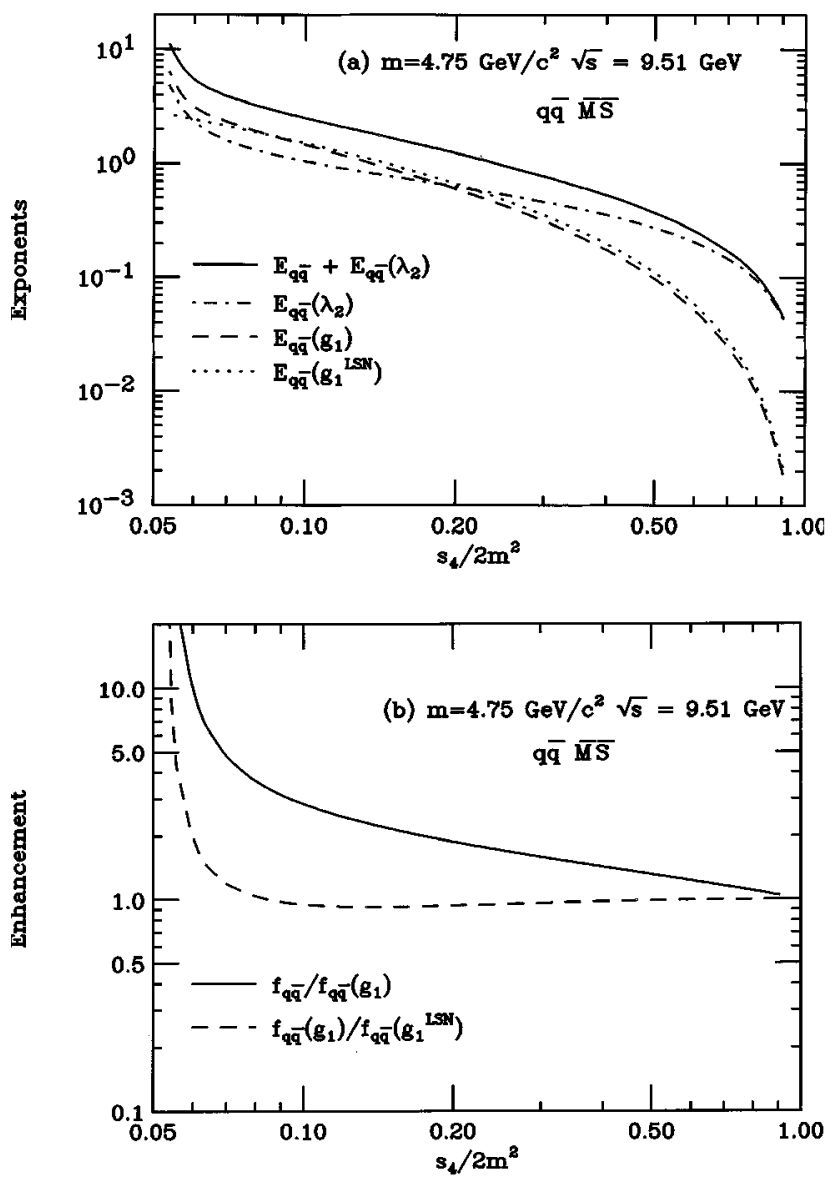

FIG. 7. We show the contributions to $f_{q \bar{q}}^{\overline{\mathrm{MS}}}$ and the enhancement factors in bottom quark production for $m=4.75 \mathrm{GeV} / c^{2}$ and $\sqrt{s}=9.51 \mathrm{GeV}$. The labels are as in Fig. 2 .

effectively changes $s_{\text {cut }}$ in Eq. (3.2) since $s_{4, \min }=2 m^{2} \Lambda / Q$. Therefore increasing $Q^{2}$ correspondingly increases the effective $s_{4}$ integration region. This latter effect seems to be the most important because the largest scale also produces the largest $g g$ contribution to the cross section when only the LL terms are considered.

When $s_{\text {cut }}=5 s_{4, \min }$, the gluon contribution is enhanced and actually dominates for $Q^{2}=m^{2}$ and $4 m^{2}$, seen in Table II. This is not surprising since at $s_{\text {cut }}=5 s_{4, \text { min }}, \mu_{0} \approx 0.1 \mathrm{~m}$ in the DIS scheme and $0.2 \mathrm{~m}$ in the $\overline{\mathrm{MS}}$ scheme when $Q^{2}=m^{2}$. At such low values of $\mu_{0}$ in the $g g$ channel, the $g g$ cross section can become large, see, e.g., Figs. 12-14 in [2]. Additionally, as $s_{\text {cut }}$ is reduced, all the contributions to the hadronic cross section increase because as $s_{\text {cut }}$ approaches $s_{4, \text { min }}$ the exponents grow more rapidly. The fastest growth of the exponents in this region occurs when $Q^{2}=m^{2} / 4$.

A comparison of the NLL and LL results in the tables helps to clarify where the NLL enhancements are largest. As discussed above, the enhancement for a fixed $s_{\text {cut }}$ is largest for $Q^{2}=4 m^{2}$ since the larger scale increases the effective $s_{4}$ probed at larger $Q^{2}$. The enhancement is also larger for lower $s_{\text {cut }}$ due to the running of the coupling constant. Finally we note that the NLL enhancement increases with mass since $t \bar{t}$ production is closer to threshold at fixed energy with larger masses. 

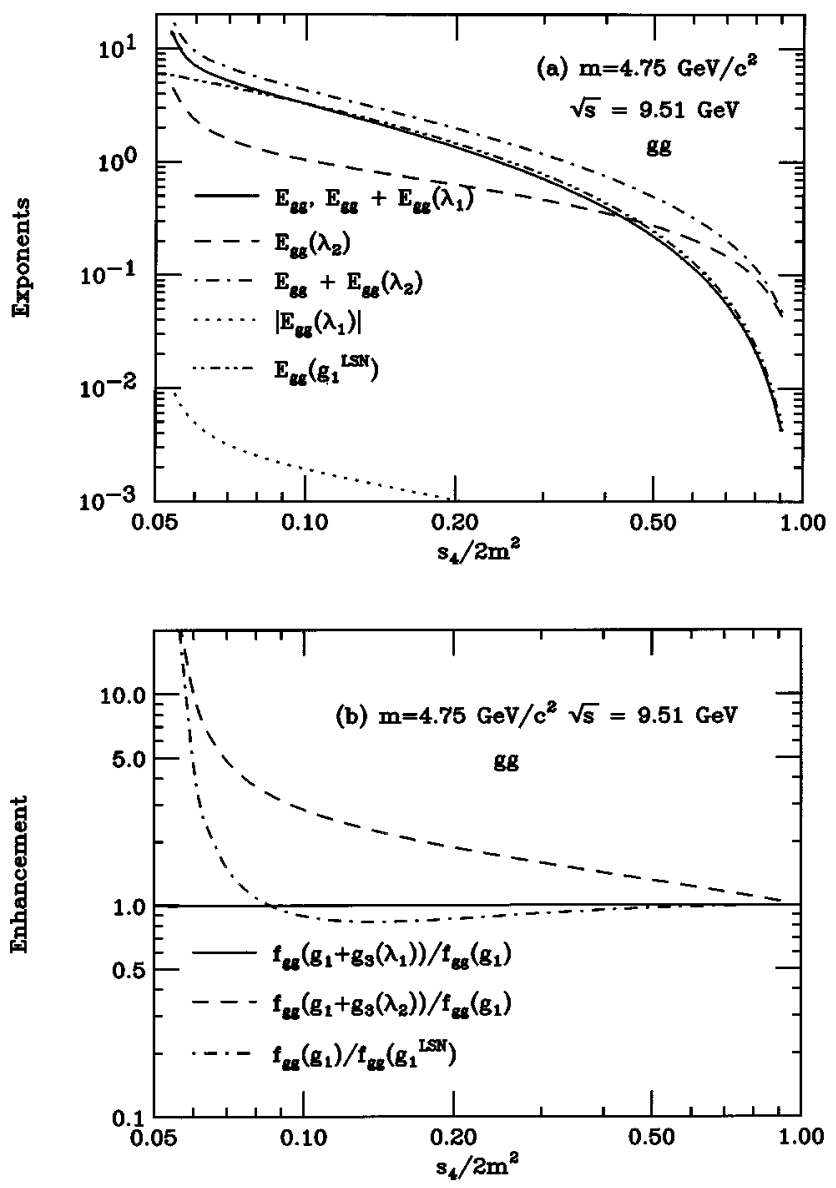

FIG. 8. We show the contributions to $f_{g g}$ and the enhancement factors in bottom quark production for $m=4.75 \mathrm{GeV} / c^{2}$ and $\sqrt{s}=9.51 \mathrm{GeV}$. The labels are as in Fig. 3 .

We conclude our discussion of top quark production by noting that the scheme dependence is rather strong, especially for lower $s_{\text {cut }}$. The scheme dependence arises because $f_{q \bar{q}}^{\overline{\mathrm{MS}}}>f_{q \bar{q}}^{\mathrm{DIS}}$, particularly near $s_{4, \mathrm{~min}}$, see Figs. 1 and 2 and also, e.g., Figs. 12 and 13 in Ref. [2]. For $s_{\text {cut }}=10 s_{4, \min }$ and $Q^{2}=m^{2}$, the $\overline{\mathrm{MS}}$ cross section is $62-71 \%$ larger than the DIS cross section at NLL and 49-55\% larger at LL. The scheme dependence is largest for small $s_{\text {cut }}$ and large $Q^{2}$ because smaller values of $s_{\text {cut }}$ are increasingly sensitive to the upper limit of the $\xi$ integral in $E_{a b}\left(g_{1}\right)$, see the Appendix. The scheme dependence increases slightly with mass at fixed energy. Thus close to threshold the scheme dependence is unavoidable. Even though the partonic cross section is only weakly scheme dependent for $\eta>1$, as seen in Fig. 4, some scheme dependence will remain until new sets of parton densities which have incorporated resummation effects before being fitted to data are available. In the absence of such densities we favor the $\overline{\mathrm{MS}}$ results as they have a more reliable theoretical basis and the $q \bar{q}$ and $g g$ channels can be treated consistently only in this scheme.

Since $\alpha_{s}$ depends strongly on the heavy quark mass scale, we repeat our calculations for bottom quark production with $m=4.75 \mathrm{GeV} / c^{2}$ at $\sqrt{s}=9.51 \mathrm{GeV}$. The smaller quark mass and the larger $\Lambda_{4}, 0.23 \mathrm{GeV}$ for the MRS $\mathrm{D}_{-}^{\prime}$ distributions, result in a reduced range in $s_{4} / 2 \mathrm{~m}^{2}$ before the exponents diverge, as shown in Figs. 6-8. The exponents tend to be
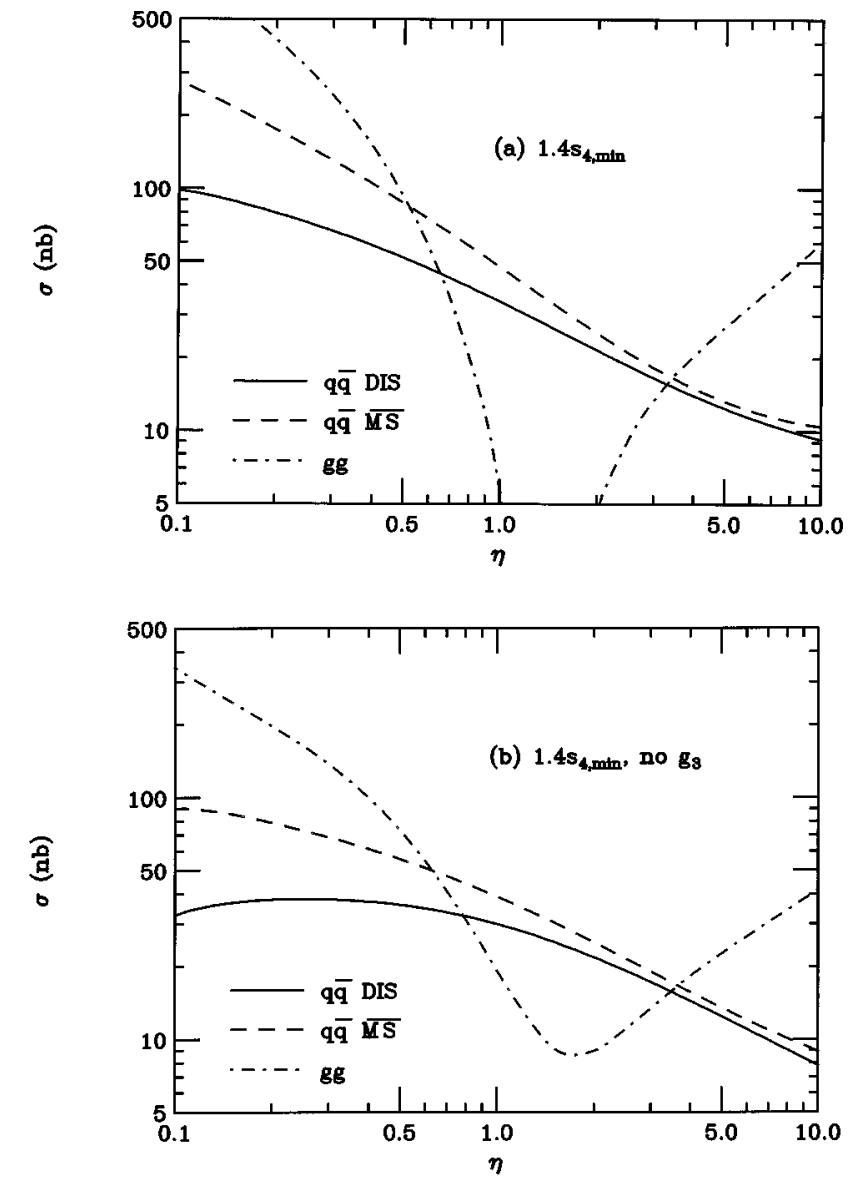

FIG. 9. The partonic bottom quark production cross section is shown as a function of $\eta$. The labels are as in Fig. 4. We use $s_{\text {cut }} \approx 1.4 s_{4, \text { min }}$ with the NLL $g_{3}$ contributions in (a) and without them in (b).

somewhat smaller for the bottom quark except near $s_{4, \min }$ due to the faster running of the coupling constant. The running of $\alpha_{s}$ also produces faster growth of the exponents at lower $Q^{2}$. Thus the scale dependence is also stronger for $b \bar{b}$ production. We note that the energy dependence of $g_{3}$ is stronger for the lighter quark mass. While $E_{g g}\left(\lambda_{1}\right)$ is smaller near threshold, it increases faster with energy than at the top quark mass. In general, the bottom quark cross section is more sensitive to $s_{\text {cut }}$ and $Q^{2}$ than the much more massive top quarks, see also $[7,8]$.

The partonic cross sections with $s_{\text {cut }}=1.4 s_{4, \text { min }}$ and $Q^{2}=m^{2}$ are shown with and without the NLL $g_{3}$ terms in Fig. 9. This value of $s_{\text {cut }}$ corresponds to $\mu_{0} \approx 0.37 \mathrm{~m}$ and $0.51 m$ in the DIS and $\overline{\mathrm{MS}}$ schemes, somewhat larger than those in $[7,8]$. Varying $s_{\text {cut }}$ we find that for $\eta<1$, the partonic cross section can change by an order of magnitude near $\eta \sim 0.1$ and a factor of 2 to 3 at $\eta \sim 0.5$. The largest variation is in the $g g$ channel. Of course the parton luminosity in the $x$ range probed at a given energy determines the sensitivity of the hadronic cross section. At higher energies, away from threshold, the sensitivity to $s_{\text {cut }}$ decreases. Similar trends can be seen in charm production although for $c \bar{c}$ production, $s_{4, \min }$ already corresponds to the $\mu_{0}$ values used in [8], suggesting that a stable cross section is even more difficult to obtain for charm production. 

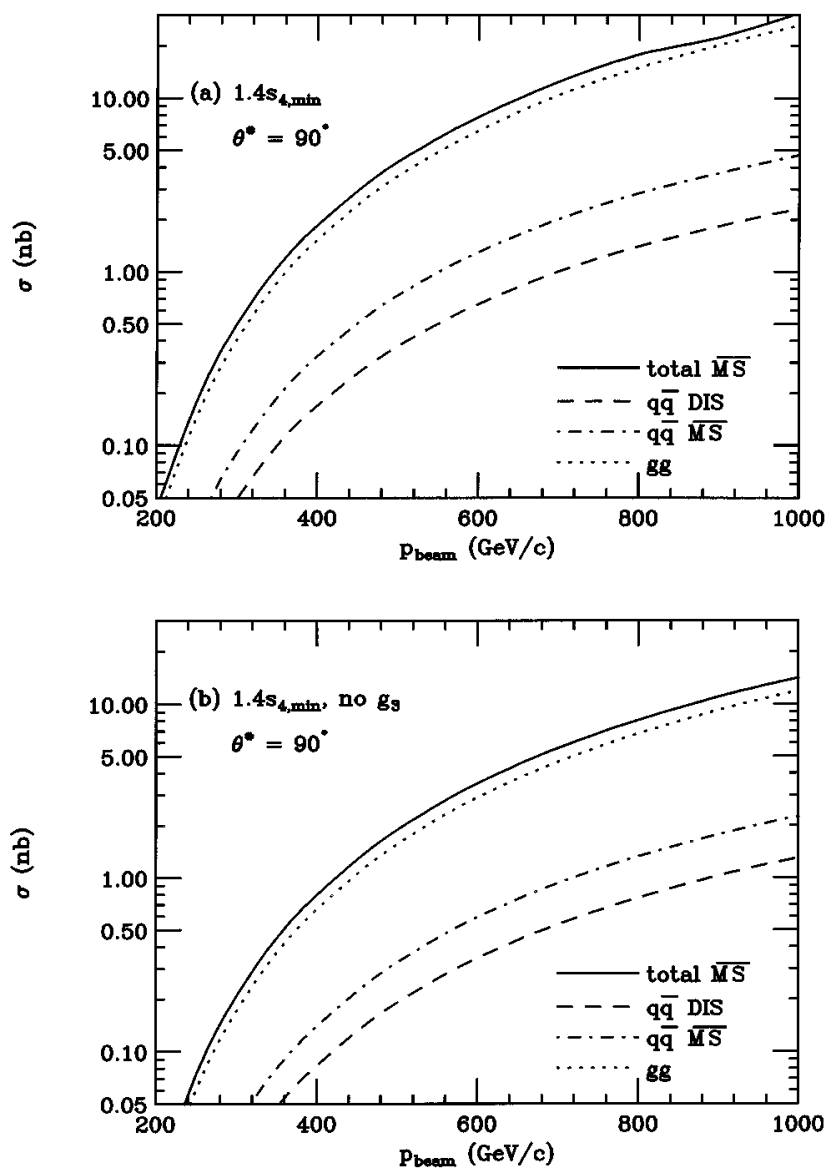

FIG. 10. The hadronic $b \bar{b}$ production cross section at $\theta^{*}=90^{\circ}$ is given as a function of beam momentum for $p p$ interactions. We use the MRS $\mathrm{D}_{-}^{\prime}$ parton densities in the DIS scheme for $q \bar{q}$ annihilation (dashed) and in the $\overline{\mathrm{MS}}$ scheme for the $q \bar{q}$ (dotdashed) and $g g$ (dotted) channels. The $\overline{\mathrm{MS}}$ sum is given in the solid curve. The results are given for $s_{\text {cut }} \approx 1.4 s_{4 \text {,min }}$ with the NLL $g_{3}$ corrections in (a) and without them in (b).

In Fig. 10 we show the $b \bar{b}$ production cross section at $\theta^{*}=90^{\circ}$ as a function of beam momentum for $p p$ interactions with $s_{\mathrm{cut}} \approx 1.4 s_{4, \min }$ and $Q^{2}=m^{2}$, with and without the NLL contributions. As expected, $g g$ fusion is dominant $[7,8]$. These energies, $20 \leqslant \sqrt{S} \leqslant 45 \mathrm{GeV}$, correspond to $3.4 \leqslant \eta_{\max } \leqslant 21.4$. At the HERA-B energy $\sqrt{S}=39.2 \mathrm{GeV}$ we find a total $\overline{\mathrm{MS}}$ cross section at $\theta^{*}=90^{\circ}$ of $8.6 \mathrm{nb}$ at $\mathrm{LL}$ and $19.1 \mathrm{nb}$ at NLL, more than a factor of 2 enhancement. Note that the $b \bar{b}$ cross section is somewhat smaller than found previously $[7,8]$, in part because we are only considering a fixed angle.

The sensitivity to $s_{\text {cut }}$ and the scale is detailed in Tables IV-VI for $s_{\text {cut }}=1.2,1.4$, and $1.6 s_{4, \min }$ and $Q^{2}=m^{2}, 4 m^{2}$, and $m^{2} / 4$. The same trends can be observed as we remarked upon for $t \bar{t}$ production although the enhancements are generally larger for $b \bar{b}$ production. We note that for the values of $s_{\text {cut }}$ chosen here, the NLL enhancement and scheme dependence decrease with increasing energy since the higher energy probes the region where the corrections are less important. There seems to be a general decrease of the scheme dependence with energy for the bottom quarks. However, it is difficult to fairly compare the scheme dependence of top quark and bottom quark production since our values of $s_{\text {cut }}$ do not correspond to the same $\mu_{0} / m$ ratio in both cases. Our previous work on LL resummation favored a fixed ratio to describe production of all heavy quarks [7,8]. Thus, while our bottom quark results are illustrative only, they clearly indicate that a full NLL calculation including all angles is needed to clarify bottom quark resummation.

\section{CONCLUSIONS}

We have investigated the numerical importance of the NLL terms in the resummation of subleading soft gluon contributions near threshold for heavy quark production. We have shown that, for the resummation method of [2] and at $\theta^{*}=90^{\circ}$ these contributions are either numerically small or there are cancellations between them. Therefore the inclusion of the NLL terms leads to only a moderate change in the top quark production cross section at $\theta^{*}=90^{\circ}$. Bottom quark production is much more sensitive to $s_{\text {cut }}$, making definitive statements about the resummed cross sections more difficult, particularly in a model without a calculable perturbative cutoff.

\section{ACKNOWLEDGMENTS}

We would like to thank G. Sterman and E. Laenen for helpful discussions. R. Vogt and N. Kidonakis would like to thank Brookhaven National Laboratory for hospitality. R. Vogt also thanks SUNY at Stony Brook for hospitality. J. Smith would like to thank the Alexander von Humboldt Stiftung for financial support during his sabbatical year at DESY. The work of N.K. was supported in part by the PPARC under Grant GR/K54601. The work of J.S. was supported in part under contract NSF 93-09888. The work of R.V. was supported in part by the Director, Office of Energy Research, Division of Nuclear Physics of the Office of High Energy and Nuclear Physics of the U. S. Department of Energy under Contract Number DE-AC03-76SF0098.

\section{APPENDIX}

Here we present the analytical results for the $g_{1}, g_{2}$, and $g_{3}$ (where appropriate) integrals. As previously discussed, we use the two-loop running coupling constant

$$
\begin{gathered}
\alpha_{s}(\xi)=\frac{1}{a \ln \xi}+\frac{b}{a} \frac{\ln (\ln \xi)}{\ln ^{2}(\xi)}, \\
a=\frac{11 C_{A}-4 T_{\mathrm{f}} n_{\mathrm{f}}}{12 \pi}, \\
b=-6 \frac{17 C_{A}^{2}-\left(6 C_{F}+10 C_{A}\right) T_{\mathrm{f}} n_{\mathrm{f}}}{\left(11 C_{A}-4 T_{\mathrm{f}} n_{\mathrm{f}}\right)^{2}},
\end{gathered}
$$

with $C_{A}=3, C_{F}=4 / 3$ and $T_{\mathrm{f}}=1 / 2$. It is obvious that when $\xi<1, \ln \xi$ is negative so that $\ln (\ln \xi)$ has a cut and needs a precise definition. We use the cutoff on the $\omega^{\prime}$ variable to stop the integration at that point.

In general, 
TABLE IV. The hadronic $b \bar{b}$ production cross sections in $p p$ interactions for $s_{\text {cut }}=1.4 s_{4, \min }, m=4.75$ $\mathrm{GeV} / c^{2}$, and $\theta^{*}=90^{\circ}$.

\begin{tabular}{|c|c|c|c|c|c|c|}
\hline \multirow[t]{2}{*}{$p_{\text {lab }}(\mathrm{GeV} / c)$} & $\sigma_{q \bar{q}}^{\mathrm{DIS}}(\mathrm{nb})$ & $\begin{array}{c}\sigma_{q \bar{q}}^{\mathrm{NLL}}(\mathrm{nb}) \\
\overline{\bar{q}}\end{array}$ & $\sigma_{g g}(\mathrm{nb})$ & $\sigma_{q \bar{q}}^{\mathrm{DIS}}(\mathrm{nb})$ & $\frac{\mathrm{LL}}{\sigma_{q \bar{q}}^{\overline{\mathrm{MS}}}(\mathrm{nb})}$ & $\sigma_{g g}(\mathrm{nb})$ \\
\hline & \multicolumn{6}{|c|}{$Q^{2}=m^{2} \Rightarrow \mu_{0}^{\mathrm{DIS}}=0.37 m, \mu_{0}^{\overline{\mathrm{MS}}}=0.51 m$} \\
\hline 200 & 0.0049 & 0.0081 & 0.034 & 0.0021 & 0.0031 & 0.014 \\
\hline 300 & 0.049 & 0.091 & 0.410 & 0.023 & 0.037 & 0.172 \\
\hline 400 & 0.169 & 0.326 & 1.54 & 0.083 & 0.140 & 0.658 \\
\hline 500 & 0.373 & 0.734 & 3.58 & 0.190 & 0.325 & 1.56 \\
\hline 600 & 0.653 & 1.30 & 6.52 & 0.344 & 0.591 & 2.90 \\
\hline 700 & 1.00 & 2.01 & 10.44 & 0.537 & 0.927 & 4.64 \\
\hline 800 & 1.40 & 2.84 & 15.00 & 0.764 & 1.32 & 6.76 \\
\hline 900 & 1.84 & 3.72 & 20.23 & 1.02 & 1.77 & 9.20 \\
\hline \multirow[t]{2}{*}{1000} & 2.31 & 4.70 & 26.18 & 1.30 & 2.25 & 11.86 \\
\hline & \multicolumn{6}{|c|}{$Q^{2}=4 m^{2} \Rightarrow \mu_{0}^{\mathrm{DIS}}=0.52 m, \mu_{0}^{\overline{\mathrm{MS}}}=0.82 m$} \\
\hline 200 & 0.0036 & 0.0079 & 0.041 & 0.0016 & 0.0029 & 0.015 \\
\hline 300 & 0.036 & 0.082 & 0.482 & 0.017 & 0.032 & 0.183 \\
\hline 400 & 0.124 & 0.286 & 1.81 & 0.060 & 0.118 & 0.702 \\
\hline 500 & 0.273 & 0.636 & 4.32 & 0.138 & 0.269 & 1.69 \\
\hline 600 & 0.479 & 1.12 & 8.01 & 0.248 & 0.484 & 3.20 \\
\hline 700 & 0.732 & 1.72 & 12.87 & 0.387 & 0.756 & 5.15 \\
\hline 800 & 1.02 & 2.41 & 18.86 & 0.551 & 1.07 & 7.60 \\
\hline 900 & 1.35 & 3.17 & 25.91 & 0.735 & 1.43 & 10.42 \\
\hline \multirow[t]{2}{*}{1000} & 1.70 & 4.01 & 33.68 & 0.941 & 1.83 & 13.69 \\
\hline & \multicolumn{6}{|c|}{$Q^{2}=m^{2} / 4 \Rightarrow \mu_{0}^{\mathrm{DIS}}=0.26 m, \mu_{0}^{\overline{\mathrm{MS}}}=0.32 m$} \\
\hline 200 & 0.0041 & 0.0054 & 0.026 & 0.0019 & 0.0022 & 0.012 \\
\hline 300 & 0.050 & 0.076 & 0.354 & 0.024 & 0.033 & 0.167 \\
\hline 400 & 0.188 & 0.300 & 1.37 & 0.096 & 0.139 & 0.665 \\
\hline 500 & 0.433 & 0.713 & 3.20 & 0.232 & 0.341 & 1.59 \\
\hline 600 & 0.783 & 1.30 & 5.78 & 0.429 & 0.640 & 2.94 \\
\hline 700 & 1.21 & 2.04 & 9.11 & 0.682 & 1.03 & 4.69 \\
\hline 800 & 1.72 & 2.92 & 12.96 & 0.985 & 1.49 & 6.79 \\
\hline 900 & 2.27 & 3.90 & 17.37 & 1.33 & 2.02 & 9.16 \\
\hline 1000 & 2.87 & 4.95 & 22.29 & 1.70 & 2.60 & 11.81 \\
\hline
\end{tabular}

$$
\begin{aligned}
E_{a b}^{\mathrm{sch}}= & \int_{\omega_{0}}^{1} \frac{d \omega^{\prime}}{\omega^{\prime}}\left(\int_{\xi_{L}}^{\xi_{U}^{\mathrm{sch}}} \frac{d \xi}{\xi}\left[\gamma_{a b}\left(\alpha_{s}(\xi)+\frac{K}{2 \pi} \alpha_{s}^{2}(\xi)\right)\right]\right. \\
& -\delta_{a b}^{\mathrm{sch}} \alpha_{s}\left(\frac{\omega^{\prime} Q^{2}}{\Lambda^{2}}\right)-\left\{\lambda_{i, a b}\left[\alpha_{s}\left(\frac{\omega^{\prime 2} Q^{2}}{\Lambda^{2}}\right), \theta^{*}=90^{\circ}\right]\right. \\
& \left.\left.+\lambda_{i, a b}^{*}\left[\alpha_{s}\left(\frac{\omega^{\prime 2} Q^{2}}{\Lambda^{2}}\right), \theta^{*}=90^{\circ}\right]\right\}\right),
\end{aligned}
$$

where $\quad \xi_{L}=\omega^{\prime 2} Q^{2} / \Lambda^{2}, \quad \xi_{U}^{\mathrm{DIS}}=\omega^{\prime} Q^{2} / \Lambda^{2}, \quad \xi_{U}^{\overline{\mathrm{MS}}}=Q^{2} / \Lambda^{2}$, $\gamma_{q \bar{q}}=2 C_{F} / \pi, \gamma_{g g}=2 C_{A} / \pi$, and $\delta_{a b}^{\mathrm{sch}}=3 C_{F} / 2 \pi$ for $q \bar{q}$ in the DIS scheme and 0 otherwise. We will give the results for single integrals over $\omega^{\prime}$ first. For convenience, we define

$$
L_{0}=\ln \left(\frac{Q^{2}}{\Lambda^{2}}\right) ; \quad L_{1}=\ln \left(\frac{\omega_{0} Q^{2}}{\Lambda^{2}}\right) ; \quad L_{2}=\ln \left(\frac{\omega_{0}^{2} Q^{2}}{\Lambda^{2}}\right)
$$

Note that the contributions dependent on $L_{2}$ set the lower limit on $s_{\text {cut }}$ since $\omega_{0} \sim \Lambda / Q$ is the lowest $\omega^{\prime}$ at which the integrals diverge.

Then, in the DIS scheme, the $g_{2}$ result is

$$
\begin{aligned}
E_{q \bar{q}}^{\mathrm{DIS}}\left(g_{2}\right)= & -\frac{3}{2} \frac{C_{F}}{\pi a}\left[\ln L_{0}-\frac{b}{L_{0}}\left(\ln L_{0}+1\right)-\ln L_{1}\right. \\
& \left.+\frac{b}{L_{1}}\left(\ln L_{1}+1\right)\right] .
\end{aligned}
$$

For the terms in $\lambda_{i, a b}$ which only contain an $s_{4}^{\prime}$ dependence in the running coupling constant, the $g_{3}$ integral can also be done analytically. In these cases,

$$
E_{a b}\left(g_{3}\right) \propto \frac{1}{2 a}\left[\ln L_{0}-\frac{b}{L_{0}}\left(\ln L_{0}+1\right)-\ln L_{2}+\frac{b}{L_{2}}\left(\ln L_{2}+1\right)\right] .
$$


TABLE V. The hadronic $b \bar{b}$ production cross sections in $p p$ interactions for $s_{\text {cut }}=1.2 s_{4, \min }, m=4.75$ $\mathrm{GeV} / c^{2}$, and $\theta^{*}=90^{\circ}$.

\begin{tabular}{|c|c|c|c|c|c|c|}
\hline \multirow[t]{2}{*}{$p_{\text {lab }}(\mathrm{GeV} / c)$} & $\sigma_{q \bar{q}}^{\mathrm{DIS}}(\mathrm{nb})$ & $\sigma_{q \bar{q}}^{\overline{\mathrm{ML}}}(\mathrm{nb})$ & $\sigma_{g g}(\mathrm{nb})$ & $\sigma_{q \bar{q}}^{\mathrm{DIS}}(\mathrm{nb})$ & $\sigma_{q \bar{q}}^{\frac{\mathrm{LL}}{\mathrm{MS}}}(\mathrm{nb})$ & $\sigma_{g g}(\mathrm{nb})$ \\
\hline & \multicolumn{6}{|c|}{$Q^{2}=m^{2} \Rightarrow \mu_{0}^{\mathrm{DIS}}=0.34 m, \mu_{0}^{\overline{\mathrm{MS}}}=0.49 m$} \\
\hline 200 & 0.0094 & 0.020 & 0.248 & 0.0029 & 0.0049 & 0.051 \\
\hline 300 & 0.087 & 0.206 & 2.75 & 0.029 & 0.055 & 0.594 \\
\hline 400 & 0.287 & 0.700 & 9.82 & 0.103 & 0.199 & 2.19 \\
\hline 500 & 0.617 & 1.54 & 22.43 & 0.232 & 0.452 & 5.06 \\
\hline 600 & 1.06 & 2.67 & 40.53 & 0.413 & 0.811 & 9.21 \\
\hline 700 & 1.59 & 4.05 & 63.18 & 0.639 & 1.26 & 14.67 \\
\hline 800 & 2.20 & 5.62 & 89.85 & 0.902 & 1.78 & 20.84 \\
\hline 900 & 2.85 & 7.32 & 120.6 & 1.20 & 2.37 & 28.00 \\
\hline \multirow[t]{2}{*}{1000} & 3.56 & 9.18 & 154.2 & 1.51 & 2.99 & 36.60 \\
\hline & \multicolumn{6}{|c|}{$Q^{2}=4 m^{2} \Rightarrow \mu_{0}^{\mathrm{DIS}}=0.48 m, \mu_{0}^{\overline{\mathrm{MS}}}=0.77 m$} \\
\hline 200 & 0.0063 & 0.017 & 0.301 & 0.0019 & 0.0042 & 0.057 \\
\hline 300 & 0.059 & 0.171 & 3.41 & 0.020 & 0.045 & 0.665 \\
\hline 400 & 0.193 & 0.575 & 12.56 & 0.071 & 0.159 & 2.51 \\
\hline 500 & 0.416 & 1.25 & 29.35 & 0.159 & 0.356 & 5.96 \\
\hline 600 & 0.716 & 2.16 & 54.00 & 0.284 & 0.635 & 11.10 \\
\hline 700 & 1.08 & 3.30 & 85.96 & 0.442 & 0.985 & 17.73 \\
\hline 800 & 1.49 & 4.58 & 125.7 & 0.626 & 1.39 & 26.18 \\
\hline 900 & 1.95 & 5.97 & 170.1 & 0.829 & 1.84 & 35.82 \\
\hline \multirow[t]{2}{*}{1000} & 2.45 & 7.47 & 220.3 & 1.05 & 2.34 & 46.82 \\
\hline & \multicolumn{6}{|c|}{$Q^{2}=m^{2} / 4 \Rightarrow \mu_{0}^{\mathrm{DIS}}=0.24 m, \mu_{0}^{\overline{\mathrm{MS}}}=0.31 m$} \\
\hline 200 & 0.0099 & 0.016 & 0.201 & 0.0030 & 0.0041 & 0.046 \\
\hline 300 & 0.106 & 0.200 & 2.35 & 0.035 & 0.056 & 0.571 \\
\hline 400 & 0.369 & 0.735 & 8.33 & 0.132 & 0.218 & 2.10 \\
\hline 500 & 0.813 & 1.66 & 18.63 & 0.306 & 0.516 & 4.80 \\
\hline 600 & 1.41 & 2.94 & 32.98 & 0.557 & 0.948 & 8.63 \\
\hline 700 & 2.14 & 4.51 & 50.25 & 0.868 & 1.49 & 13.36 \\
\hline 800 & 2.97 & 6.33 & 70.60 & 1.24 & 2.13 & 19.03 \\
\hline 900 & 3.85 & 8.26 & 92.73 & 1.65 & 2.85 & 25.16 \\
\hline 1000 & 4.78 & 10.32 & 116.5 & 2.10 & 3.63 & 32.12 \\
\hline
\end{tabular}

Thus the $g_{3}$ contributions diverge at a larger value of $\omega_{0}$ than do the $g_{2}$.

After the $\xi$ integral, the $g_{1}$ exponent is

$$
\begin{aligned}
E_{a b}^{\mathrm{sch}}\left(g_{1}\right)= & \frac{\gamma_{a b}}{a} \int_{\omega_{0}}^{1} \frac{d \omega^{\prime}}{\omega^{\prime}}\left[\left\{\ln (\ln \xi)-\frac{b}{\ln \xi}[\ln (\ln \xi)+1]\right\}-\frac{K}{2 \pi a \ln \xi}\left\{1+\frac{b}{2 \ln \xi}[2 \ln (\ln \xi)+1]\right.\right. \\
& \left.\left.+\frac{b^{2}}{27 \ln ^{2} \xi}\left[9 \ln ^{2}(\ln \xi)+6 \ln (\ln \xi)+2\right]\right\}\right]\left.\right|_{\xi_{L}} ^{\xi_{U}^{\mathrm{sch}}} .
\end{aligned}
$$

In the DIS scheme the final result is

$$
\begin{aligned}
E_{a b}^{\mathrm{DIS}}\left(g_{1}\right)= & \frac{\gamma_{a b}}{a}\left[\frac{1}{2} L_{0}\left(\ln L_{0}-1\right)-\frac{b}{4} \ln L_{0}\left(\ln L_{0}+2\right)-L_{1}\left(\ln L_{1}-1\right)+\frac{b}{2} \ln L_{1}\left(\ln L_{1}+2\right)+\frac{1}{2} L_{2}\left(\ln L_{2}-1\right)-\frac{b}{4} \ln L_{2}\left(\ln L_{2}+2\right)\right. \\
& -\frac{K}{2 \pi a}\left\{\frac{1}{2} \ln L_{0}-\frac{b}{4 L_{0}}\left(2 \ln L_{0}+3\right)-\frac{b^{2}}{216 L_{0}^{2}}\left(18 \ln ^{2} L_{0}+30 \ln L_{0}+19\right)-\ln L_{1}+\frac{b}{2 L_{1}}\left(2 \ln L_{1}+3\right)\right. \\
& \left.\left.+\frac{b^{2}}{108 L_{1}^{2}}\left(18 \ln ^{2} L_{1}+30 \ln L_{1}+19\right)+\frac{1}{2} \ln L_{2}-\frac{b}{4 L_{2}}\left(2 \ln L_{2}+3\right)-\frac{b^{2}}{216 L_{2}^{2}}\left(18 \ln ^{2} L_{2}+30 \ln L_{2}+19\right)\right\}\right] .
\end{aligned}
$$

The result is somewhat simpler in the $\overline{\mathrm{MS}}$ scheme since $\xi_{U}^{\overline{\mathrm{MS}}}$ does not depend on $\omega^{\prime}$. In this case, 
TABLE VI. The hadronic $b \bar{b}$ production cross sections in $p p$ interactions for $s_{\text {cut }}=1.6 s_{4, \min }, m=4.75$ $\mathrm{GeV} / c^{2}$, and $\theta^{*}=90^{\circ}$.

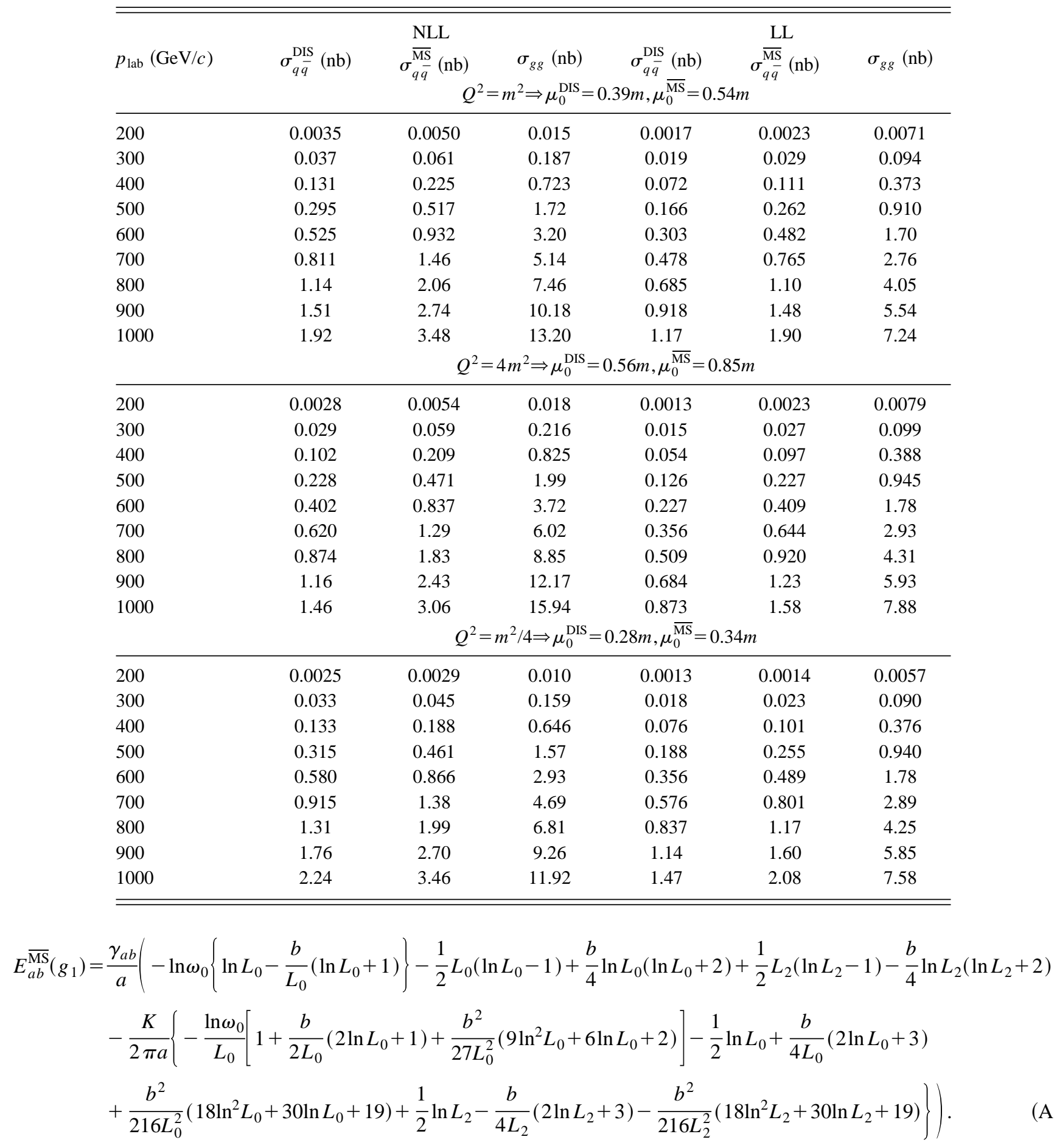

[1] G. Sterman, Nucl. Phys. B281, 310 (1987); S. Catani and L. Trentadue, ibid. B327, 323 (1989).

[2] E. Laenen, J. Smith, and W.L. van Neerven, Nucl. Phys. B369, 543 (1992).

[3] E. Laenen, J. Smith, and W.L. van Neerven, Phys. Lett. B 321, 254 (1994).

[4] CDF Collaboration, F. Abe et al., Phys. Rev. Lett. 74, 2626
(1995); D0 Collaboration, S. Abachi et al., ibid. 74, 2632 (1995).

[5] E.L. Berger and H. Contopanagos, Phys. Lett. B 361, 115 (1995); Phys. Rev. D 54, 3085 (1996); Report No. ANL-HEPCP-96-51, hep-ph/9606421 (unpublished).

[6] S. Catani, M.L. Mangano, P. Nason, and L. Trentadue, Nucl. Phys. B478, 273 (1996); Phys. Lett. B 378, 329 (1996). 
[7] N. Kidonakis and J. Smith, Mod. Phys. Lett. A 11, 587 (1996).

[8] J. Smith and R. Vogt, Z. Phys. C (to be published).

[9] W.L. van Neerven and E.B. Zijlstra, Nucl. Phys. B382, 11 (1992); T. Matsuura, R. Hamburg, and W.L. van Neerven, ibid. B359, 343 (1991).

[10] R. Meng, G.A. Schuler, J. Smith, and W.L. van Neerven, Nucl. Phys. B339, 325 (1990).

[11] N. Kidonakis and G. Sterman, Phys. Lett. B 387, 867 (1996); contribution presented at the Les Rencontres de Physique de la Vallée d'Aoste, La Thuile, Aosta Valley, 1996, Report No. hep-ph/9607222 (unpublished).

[12] N. Kidonakis, Report No. hep-ph/9606474, UMI-96-36628-mc (microfiche), 1996 (unpublished); N. Kidonakis and G. Sterman, Report No. EDINBURGH 97/3, ITP-SB-97-24, 1997 (unpublished).

[13] J.C. Collins, D.E. Soper, and G. Sterman, in Perturbative
Quantum Chrodynamics, edited by A.H. Mueller (World Scientific, Singapore, 1989), p. 1.

[14] J.G.M. Gatheral, Phys. Lett. 133B, 90 (1983); J.C. Collins, in Perturbative Quantum Chrodynamics [13], p. 573.

[15] H. Contopanagos, E. Laenen, and G. Sterman, Nucl. Phys. B484, 303 (1997).

[16] J. Kodaira and L. Trentadue, Phys. Lett. 112B, 66 (1982).

[17] G. Sterman (private communication).

[18] A.D. Martin, R.G. Roberts, and W.J. Stirling, Phys. Lett. B 306, 145 (1993).

[19] H. Plothow-Besch, PDFLIB: Nucleon, Pion and Photon Parton Density Functions and $\alpha_{s}$ Calculations, User's ManualVersion 4.16, Report No. W5051 PDFLIB, 1994.01.11, CERN-PPE (unpublished); Comput. Phys. Commun. 75, 396 (1993). 